\title{
Master Equation Study of Vibrational and Rotational Relaxation of Oxygen
}

\author{
Daniil A. Andrienko* \\ Iain D. Boyd ${ }^{\dagger}$ \\ Department of Aerospace Engineering, University of Michigan, Ann Arbor, MI, 48109
}

\begin{abstract}
A complete set of state-to-state transition cross sections for $\mathrm{O}_{2}-\mathrm{O}$ is generated by the quasi-classical trajectory method on an accurate three-body potential energy surface. Transition rates obtained by integrating the cross section data are employed in the system of master equations to study trans-rotational and trans-vibrational energy exchanges in a wide range of heat bath conditions. The energy-equivalent definition of temperature is adopted to obtain characteristic relaxation times. It is shown that the vibrational relaxation becomes less efficient at high temperatures, in contrast with the conventional equation for relaxation time, proposed by Millikan and White. Rotational and vibrational relaxation times are the same order of magnitude in the range of temperatures observed in hypersonic flows.
\end{abstract}

\section{Introduction}

During flight at hypersonic speed, a significant degree of thermal nonequilibrium in the flow field can be observed. At velocities typical for reentry capsules and hypersonic vehicles, the nonequilibrium excitation of internal degrees of freedom can take place in a shock wave. Because oxygen quickly dissociates during re-entry, the $\mathrm{O}_{2}$ thermochemistry has attained less attention than other aspects of hypersonic aerothermodynamics. However, during flight at moderate velocities, a significant amount of molecular oxygen can be encountered in the post-shock region. Meanwhile, significant progress in the computational simulation of atmospheric species has been achieved over the past several decades. Toward this end, the application of advanced numerical models for the simulation of oxygen chemistry is described in the current work.

Nowadays, the Quasi-Classical Trajectory (QCT) method is used to generate cross section data for important three-body systems such as $\mathrm{N}_{2}-\mathrm{N}, \mathrm{N}_{2}-\mathrm{O}$ and $\mathrm{H}_{2}-\mathrm{H}$. In these calculations the simulation of atommolecule collisions with randomly sampled parameters is preceded by a choice of an accurate, preferably ab-initio, potential energy surface (PES), which ultimately governs the properties of molecular systems.

Further analysis involves the solution of kinetic equations that are coupled to the set of state-specific transition rates, obtained through the integration of cross sections. This step presents a significant computational challenge due to the large number of rovibrational levels. An accurate state-to-state model considers each energy state as a separate species. The total number of equations is on the order of several thousand for each type of molecule-atom collision, and such systems of equations are numerically stiff due to the rapid dissociation at high temperatures.

These factors make the state-to-state kinetic approach an extremely expensive and, at the same time, a very desirable technique, since it is possible to model the thermal nonequilibrium in a shock flow without assuming the existence of internal temperature. Recent works in this direction have reported the studies of rovibrational relaxation and dissociation in the $\mathrm{N}_{2}-\mathrm{N}[1,2]$ and the $\mathrm{O}_{2}-\mathrm{Ar}$ [3] systems under constant heat bath conditions. Among other molecular systems, the interaction of $\mathrm{O}_{2}$ with its parent atom is of interest [4]. Oxygen chemistry is also important in the flight environment of scramjet engines. On the other hand, vibrational relaxation in $\mathrm{O}_{2}-\mathrm{O}$ collisions is known to proceed several orders of magnitude faster than that of other molecules [5] due to the large attractive component in the $\mathrm{O}_{3}$ potential energy surface [6].

\footnotetext{
*Postdoctoral research fellow, Department of Aerospace Engineering, University of Michigan, 1320 Beal Ave

$\dagger$ James E. Knott Professor, Department of Aerospace Engineering, University of Michigan, 1320 Beal Ave
} 
In the present paper, the system of master equations is employed to describe the rotational and vibrational relaxation in $\mathrm{O}_{2}-\mathrm{O}$ collisions utilizing the state-specific transition rates, computed by the QCT method. Two potential energy surfaces are adopted to investigate the level of fidelity required to build an accurate thermodynamic model. This paper is organized as follows. Section II provides a background on the molecular structure of oxygen and on the existing data of $\mathrm{O}_{2}-\mathrm{O}$ collisional dynamics. Section III addresses the governing relations of the QCT method and master equations. The detailed study of rovibrational relaxation is presented in Section IV. Conclusions are given in Section V. The supplemental data is listed in Section VII.

\section{Background}

\section{A. $\mathrm{O}_{2}$ and $\mathrm{O}_{3}$ molecular structure}

In the present work, two potential energy surfaces of different fidelity are adopted to study the $\mathrm{O}_{3}$ system. The first PES is obtained by the summation of interactions between each pair of atoms. This potential surface does not account for the many-body interaction, however, the QCT simulation with this PES can demonstrate the influence of the three-body repulsion term. The simple potential surface is calculated as follows:

$$
V_{O_{3}}\left(r_{A B} ; r_{B C} ; r_{A C}\right)=V_{O_{2}, A B}\left(r_{A B}\right)+V_{O_{2}, B C}\left(r_{B C}\right)+V_{O_{2}, A C}\left(r_{A C}\right),
$$

where the diatomic interaction, $V_{O_{2}}(r)$, is given by the Hulburt-Hirschfelder $(\mathrm{H}-\mathrm{H})$ potential:

$$
V_{O_{2}}(r)=D\left[\left(1-\exp \left(-a\left(r-r_{e}\right)\right)\right)^{2}+c a^{3}\left(r-r_{e}\right)^{3} \exp \left(-2 a\left(r-r_{e}\right)\right)\left(1+a b\left(r-r_{e}\right)\right)\right] .
$$

Coefficients $a, b$ and $c$ are determined by the spectroscopic constants of oxygen, listed in [7]. The simple PES supports 36 vibrational states, a maximum of 231 rotational states and a total number of 5117 rovibrational states of $\mathrm{O}_{2}\left(X^{3} \Sigma_{g}^{-}\right)$.

An accurate many-body $\mathrm{O}_{3}$ PES [6] is also adopted for the purpose of high fidelity simulation. This PES is obtained by the double many body expansion method and contains two- and three-body interaction terms. The potential energy surface by Varandas generates 47 vibrational levels and a maximum of 236 rotational levels for molecular oxygen in the ground electronic state. The total number of rovibrational states is equal to 6,245 , however, taking into account the nuclear spin statistics of a homonuclear molecule, the even numbered rotational levels for the $\mathrm{O}_{2}\left(X^{3} \Sigma_{g}^{-}\right)$state are forbidden. Since, during a bound-bound rovibrational transition, the symmetry of the initial and final states cannot change, transitions of the $\mathrm{O}_{2}$ molecule in the ground electronic state are only allowed between odd-numbered rotational levels [8].

When incorporated into the QCT code, the Varandas PES requires approximately 8 to 10 times more computational resources than the simple HH PES. Due to this reason, calculation of state-specific transition rates for the entire rovibrational ladder using the Varandas PES is now in progress and not reflected in the present paper. As the first step of investigation using the high fidelity PES, the rotationally-averaged transition rates for all vibrational states are obtained at rotational temperatures of 1,000, 2,000, 3,000, $5,000,10,000$ and 20,000 K. For the HH PES a complete set of rovibrational cross sections is generated and discussed in the present paper.

The comparison of $\mathrm{HH}$ and Varandas potentials is shown in Fig. 1 for the $\mathrm{O}_{3}$ collinear configuration. The HH PES suggests a deep triangular minimum of potential energy, while the PES by Varandas and Pais has a linear direction of the closest approach and two saddle points at distances of 1.6 and $2.4 \AA$. The potential barriers, introduced by these saddle points, are 0.6 and $1.9 \mathrm{eV}$ respectively. Thus, it is expected that transitions rates obtained using the HH PES will be higher than those obtained with the Varandas potential.

\section{B. Previous experimental and theoretical studies}

The vibrational relaxation of air species is usually described by the Millikan-White's equation that connects the vibrational relaxation time to the macroscopic parameters, such as temperature and pressure. In the original work [9], the analysis of a large amount of experimental data was performed. It was found that vibrational relaxation becomes more efficient as gas temperature increases. Typical relaxation times of nitrogen and oxygen species vary from $10^{-3}$ to $10^{-7} \mathrm{Atm} \times \mathrm{s}$ in the range of temperatures from 1,000 $\mathrm{K}$ to 


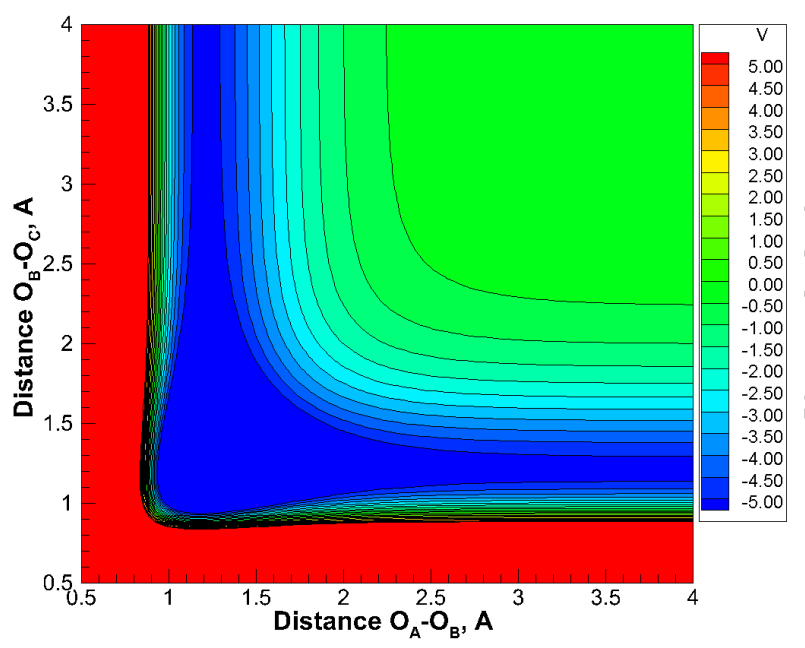

(a) Hulburt-Hirshfelder

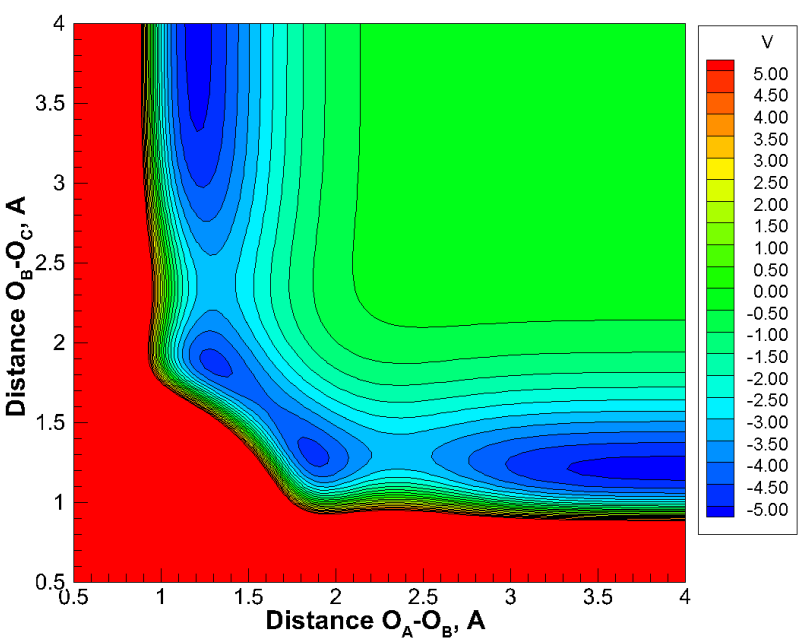

(b) Varandas and Pais

Fig. 1: $\mathrm{O}_{3}$ potential energy surface in collinear configuration

8,000 K. However, there are several exceptions to this rule, when the vibrational relaxation of some species proceeds much faster. For example, the relaxation in NO-NO, $\mathrm{O}_{2}-\mathrm{O}$ and $\mathrm{N}_{2}-\mathrm{O}$ collisions occurs two to three orders of magnitude faster than in other types of collisions. The experimental data on such molecular systems is scarce [10-12] and covers only a narrow temperature range below 4,000 $\mathrm{K}$.

The vibrational relaxation time in $\mathrm{O}_{2}-\mathrm{O}$ collisions at high temperatures was measured in experiments only by Kiefer and Lutz [13] and Breen et al. [12] using two different techniques. In the former work, atomic oxygen was generated prior to the vibrational relaxation by the thermal decomposition of ozone. Later, Breen measured the "Napier" vibrational relaxation time [14] in the shock relaxation zone. In this case, a $\mathrm{RF}$ discharge was used to generate atomic oxygen in the $\mathrm{O}_{2}-\mathrm{Ar}$ mixture and in pure $\mathrm{O}_{2}$. Both works have reported similar results, however, a substantially different temperature dependence of vibrational relaxation time was found.

According to [13], fast vibrational relaxation in $\mathrm{O}_{2}-\mathrm{O}$ collisions is the evidence of a strong chemical effect between the target and projectile particles. Breen [12] and Breig [15] suggested that the efficient energy exchange is due to the large attractive component in the three-body potential. Quack and Troe [5] employed the statistical adiabatic channel model and confirmed these results. Unfortunately, due to the lack of computational resources at that time, the investigation of $\mathrm{O}_{2}-\mathrm{O}$ collision dynamics was performed in a limited manner, leaving the problem of accurate state-to-state thermodynamic model unaddressed.

The analysis of microscopic parameters, such as collision duration and number of interatomic vibrations in the $\mathrm{O}_{2}-\mathrm{O}$ interaction, was performed in [16]. It was shown that an efficient energy exchange occurs via the strongly coupled complex formed between the projectile and target particles. The randomization of vibrational energy takes place at kinetic energies as low as $0.05 \mathrm{eV}$, particularly because of the deep potential well in the intermediate $\mathrm{O}_{3}$ molecule. At larger collision energies, vibrationally inelastic collisions are less probable. The approach, similar to [16], was adopted in the companion paper [17] to analyze the vibrational deactivation rate at different kinetic temperatures. It was found that the vibrational deactivation is more efficient at low temperatures $(500-2,000 \mathrm{~K})$ rather than at the high temperature limit. The present paper investigates of $\mathrm{O}_{2}-\mathrm{O}$ collisions under heat bath conditions by solving the master equation for each rovibrational state.

\section{Governing equations}

\section{A. QCT method}

A trajectory of target molecule and projectile atom is described by a system of first order partial differential equations: 


$$
\left\{\begin{array}{l}
\dot{q}_{i}=\frac{\partial H}{\partial p_{i}} \\
\dot{p}_{i}=-\frac{\partial H}{\partial q_{i}}, i=1,2,3,
\end{array}\right.
$$

where $q=\left\{q_{i}, Q_{i}\right\}$ are the generalized coordinates, $p=\left\{p_{i}, P_{i}\right\}$ are the conjugate momenta of the molecule and atom-molecule system, respectively, $H$ is the total Hamiltonian [18]. The continuous range of translational energy from $10^{-3}$ to $18 \mathrm{eV}$ is studied. Stratified sampling is applied to integrate the transition probability over the impact parameter. The size of the bin is $2 \times 10^{3}$ trajectories per impact parameter per collision energy. An error analysis is performed for each trajectory by comparing the initial and final total energies. The initial rovibrational state of the target molecule is assigned to a constant for each batch when computing the bound-bound rovibrational transition probability. In the case when only trans-vibrational cross sections are of interest, the initial rotational state is chosen randomly according to the Boltzmann distribution. The internuclear distance, $r_{B C}$, is sampled according to the approach, proposed in [19]. Solution of Eq. (3) is obtained by the $11^{\text {th }}$-order accurate Adams-Bashfort-Moulton method [20] with a variable time step. An averaged probability, $\bar{\Phi}$, of the bound-bound transition from the initial state $\left(\nu_{i}, j_{i}\right)$ at translational energy $E_{c o l}$ is computed as follows:

$$
\bar{\Phi}\left(E_{c o l}, \nu_{i}, j_{i}\right)=\frac{1}{(2 \pi)^{3} b_{\text {max }}^{2}} \int_{b=0}^{b_{\max }} \int_{\theta=0}^{\pi} \int_{\varphi=0}^{2 \pi} \int_{\eta=0}^{2 \pi} \int_{\xi=0}^{2 \pi} b \Phi\left(E_{c o l}, b, \theta, \varphi, \eta, \xi\right) \sin (\theta) d b d \theta d \varphi d \eta d \xi,
$$

where $\left(\nu_{i}, j_{i}\right)$ are the initial vibrational and rotational quantum numbers, the transition probability, $\Phi$, is sampled over the impact parameter $b$, orientation angles $\theta$ and $\varphi$, orientation $\eta$ of the angular momentum and initial vibrational phase angle $\xi$ of the target molecule, and $b_{\max }$ is the impact parameter at which only elastic collisions occur. The cross section of the bound-bound transition $\left(\nu_{i}, j_{i}\right) \rightarrow\left(\nu_{f}, j_{f}\right)$ is calculated as follows:

$$
\sigma\left(E_{c o l}, \nu_{i}, j_{i} \rightarrow \nu_{f}, j_{f}\right)=\pi b_{\max }^{2} \bar{\Phi}\left(E_{c o l}, \nu_{i}, j_{i} \rightarrow \nu_{f}, j_{f}\right),
$$

where $\left(\nu_{f}, j_{f}\right)$ are the final vibrational and rotational quantum numbers. Sometimes it is convenient to perform analysis assuming trans-rotational equilibrium. In this case, the state-specific cross section, given by Eq. (5), should be summed over all rotational states of the final vibrational state. The cross section of the transition $\left(\nu_{i}, j_{i}\right) \rightarrow \nu_{f}$ is derived as follows:

$$
\sigma\left(E_{c o l}, \nu_{i}, j_{i} \rightarrow \nu_{f}\right)=\sum_{j_{f}} \sigma\left(E_{c o l}, \nu_{i}, j_{i} \rightarrow \nu_{f}, j_{f}\right)
$$

Now, the cross section of the $\nu_{i} \rightarrow \nu_{f}$ transition is found by averaging the cross section in Eq. (6) over the initial rotational state, assuming that they are populated according to the Boltzmann distribution with the rotational temperature equal to the kinetic temperature:

$$
\sigma\left(E_{c o l}, \nu_{i} \rightarrow \nu_{f}\right)=\frac{\sum_{j_{i}} g_{i} \exp \left(-\left(e_{v_{i}, j_{i}}-e_{v_{i}, 0}\right) / k_{B} T\right) \sigma\left(E_{c o l}, \nu_{i}, j_{i} \rightarrow \nu_{f}\right)}{\sum_{j_{i}} g_{i} \exp \left(-\left(e_{v_{i}, j_{i}}-e_{v_{i}, 0}\right) / k_{B} T\right)},
$$

where $g_{i}=\left(2 j_{i}+1\right) \bmod \left(j_{i}, 2\right)$ is the rotational degeneracy of the state $\left(\nu_{i}, j_{i}\right)$ in the $\mathrm{O}_{2}$ ground electronic state. The rotationally-averaged rate of transition $\nu_{i} \rightarrow \nu_{f}$ is calculated as follows:

$$
K\left(T, \nu_{i} \rightarrow \nu_{f}\right)=\frac{8 \pi}{\sqrt{\mu_{A, B C}}}\left(2 \pi k_{B} T\right)^{-3 / 2} \int_{E_{c o l}=0}^{\infty} \sigma\left(E_{c o l}, \nu_{i} \rightarrow \nu_{f}\right) E_{c o l} \exp \left(\frac{-E_{c o l}}{k_{B} T}\right) d E_{c o l} .
$$

The rate of rotationally-resolved bound-bound transition $\left(\nu_{i}, j_{i}\right) \rightarrow\left(\nu_{f}, j_{f}\right)$ can be calculated in a manner similar to Eq. (8):

$$
K\left(T, \nu_{i}, j_{i} \rightarrow \nu_{f}, j_{f}\right)=\frac{8 \pi}{\sqrt{\mu_{A, B C}}}\left(2 \pi k_{B} T\right)^{-3 / 2} \int_{E_{c o l}=0}^{\infty} \sigma\left(E_{c o l}, \nu_{i}, j_{i} \rightarrow \nu_{f}, j_{f}\right) E_{c o l} \exp \left(\frac{-E_{c o l}}{k_{B} T}\right) d E_{c o l} .
$$

The principle of detailed balance is invoked to obtain the rates of endothermic bound-bound transitions in 
order to reduce the statistical error:

$$
K\left(T, \nu_{f}, j_{f} \rightarrow \nu_{i}, j_{i}\right)=K\left(T, \nu_{i}, j_{i} \rightarrow \nu_{f}, j_{f}\right) \frac{Q_{i}(T)}{Q_{f}(T)},
$$

where $Q_{i}(T)$ is the rovibrational partition function of the state $\left(\nu_{i}, j_{i}\right)$, evaluated at equilibrium temperature $T$. The reaction rate, given by Eq. (9), has to be modified to account for the degeneracy of reactants and PES. For the bound-bound transition, induced by the collision of $\mathrm{O}\left({ }^{3} P\right)$ and $\mathrm{O}_{2}\left({ }^{3} X_{g}^{-}\right)$, the degeneracy factor due to the angular momentum can be expressed in the form, given in [21]:

$$
\frac{1}{g_{B B}}=3\left(5+3 \exp \left(-\frac{227.6}{T}\right)+\exp \left(-\frac{325.9}{T}\right)\right),
$$

where the factor of 3 corresponds to the $\mathrm{O}_{2}$ triplet state. The expression in parentheses describes the degeneracy of $\mathrm{O}\left({ }^{3} P_{J}\right), J=0,1$ and 2 . Now, the degeneracy factor of the bound-bound transition is found as a ratio of the PES degeneracy to the degeneracy of reactants. Taking into account that the PES of $\mathrm{O}_{3}$ in the ground electronic state is non-degenerate, the total degeneracy is given by Eq. (11). At high temperatures, the degeneracy factor asymptotically approaches a value of $1 / 27$.

\section{B. Master equations}

At low kinetic temperatures, the population of rovibrational states converges to equilibrium significantly faster than the dissociation process takes place. At higher temperatures, dissociation and vibrational relaxation occur within the same time scale, and these processes have to be studied in a coupled manner. In the present paper, vibrational relaxation is studied in the range of kinetic temperatures ranging from 500 to $20,000 \mathrm{~K}$. Strictly speaking, the investigation at the upper limit of this range should include the molecular depletion, however, this part has been left for the future. Omitting the dissociation and recombination processes, the vibrational and rotational relaxation of oxygen can be studied by considering only bound-bound transitions. The resulting master equation for the number density of rovibrational level $i$ can be written as follows:

$$
\frac{d n_{i}}{d t}=\sum_{f \neq i}\left(K\left(T, \nu_{i}, j_{i} \rightarrow \nu_{f}, j_{f}\right) n_{O} n_{f}-K\left(T, \nu_{f}, j_{f} \rightarrow \nu_{i}, j_{i}\right) n_{O} n_{i}\right), i=1 \ldots N_{s}, f=1 \ldots N_{s}
$$

where $N_{s}$ is the total number of rovibrational states. The rates of bound-bound transitions are given by Eq. (9). Eq. (12) describes the trans-rotational-vibrational energy transfer and is termed as the RVT model. An implicit method of third order accuracy for diagonal and second order accuracy for off-diagonal elements is applied to integrate Eq. (12). The initial number density, given by $n_{O_{2}, 0}+n_{O, 0}$, is set to $1 \times 10^{18} \mathrm{~cm}^{-3}$. This is equivalent to a pressure of $1.3626 \mathrm{Atm}$ at gas temperature of 10,000 K. No mass exchange with the surrounding medium is allowed. To simulate conditions when the atom-molecule collisions are dominant, the initial number density of atomic oxygen is set to $\alpha=0.99$ of the total number density. At initial nonequilibrium conditions the population of rovibrational states is given by the Boltzmann distribution with the temperature $T_{0}=100 \mathrm{~K}$, as follows from Eq. (13):

$$
n_{i, 0}=\frac{Q_{i}\left(T_{r o t}, T_{v i b}\right)}{\sum_{i} Q_{i}\left(T_{r o t}, T_{v i b}\right)} n_{O_{2}, 0}
$$

where $Q_{i}\left(T_{\text {rot }}, T_{v i b}\right)=\left(2 j_{i}+1\right) \exp \left[-\left(e_{\nu_{i}, j_{i}}-e_{\nu_{i}, 0}\right) / k_{B} T_{\text {rot }}\right] \exp \left[-e_{\nu_{i}, 0} / k_{B} T_{v i b}\right]$ is the two-temperature rovibrational partition function, $n_{O_{2}, 0}$ is the total number density of oxygen. The initial internal temperatures, $\mathrm{T}_{r o t, 0}$ and $\mathrm{T}_{v i b, 0}$, are equal to $T_{0}$ when the RVT energy transfer is studied.

The system of governing equations in the case of trans-rotational equilibrium has the following appearance:

$$
\frac{d n_{i}}{d t}=\sum_{f \neq i}\left(K\left(T, \nu_{f} \rightarrow \nu_{i}\right) n_{O} n_{f}-K\left(T, \nu_{i}, \rightarrow \nu_{f}\right) n_{O} n_{i}\right), i=1 \ldots N_{\nu}, f=1 \ldots N_{\nu},
$$

where $N_{\nu}$ is the total number of vibrational states, transition rates are given by Eq. (8). Eq. (14) describes the trans-vibrational energy transfer and is termed as the VT model. The initial rotational temperature is 
set to the heat bath temperature, the initial vibrational temperature is set to $T_{0}$.

\section{Results}

\section{A. Verification of VT rates}

The mono- and multiquantum vibrational deactivation rates, averaged at the rotational temperature $T_{\text {rot }}=T$ in the range between 1,000 and 10,000 K, are shown in Figs. 2a and 2b, respectively. Monoquantum transition rates from $\nu_{i}=1,10$ and 20, obtained using the HH PES, are given by solid lines. The rates, obtained by the Varandas PES are shown with symbols, rates from [4] are given by dashed lines. Similar notation is adopted in Fig. 2b for transitions $\nu_{i}=10$ and 20 with $\Delta \nu=10$ and $\nu_{i}=30$ and 40 with $\Delta \nu=30$.

The difference between the $\mathrm{HH}$ and Varandas rates is the largest at low temperatures. As expected, the influence of the three-body interaction term is the most significant under these conditions. The discrepancy diminishes at high temperatures. The maximum difference is on the order of 2 to 4 times at a temperature of $1,000 \mathrm{~K}$.

The present VT rates, obtained using the Varandas PES, are in a very good agreement with those reported in [4]. One can see a disagreement at the high temperature limit $(T=10,000 \mathrm{~K})$, which is explained by the insufficient upper limit of energy scale adopted in the Esposito's calculations. In the original paper [4], the maximum of kinetic energy was set to $3 \mathrm{eV}$, while in the present work, calculations at $T=10,000 \mathrm{~K}$ are conducted up to $6.5 \mathrm{eV}$. The overall discrepancy due to the narrow energy scale reaches a maximum of 18 $\%$.

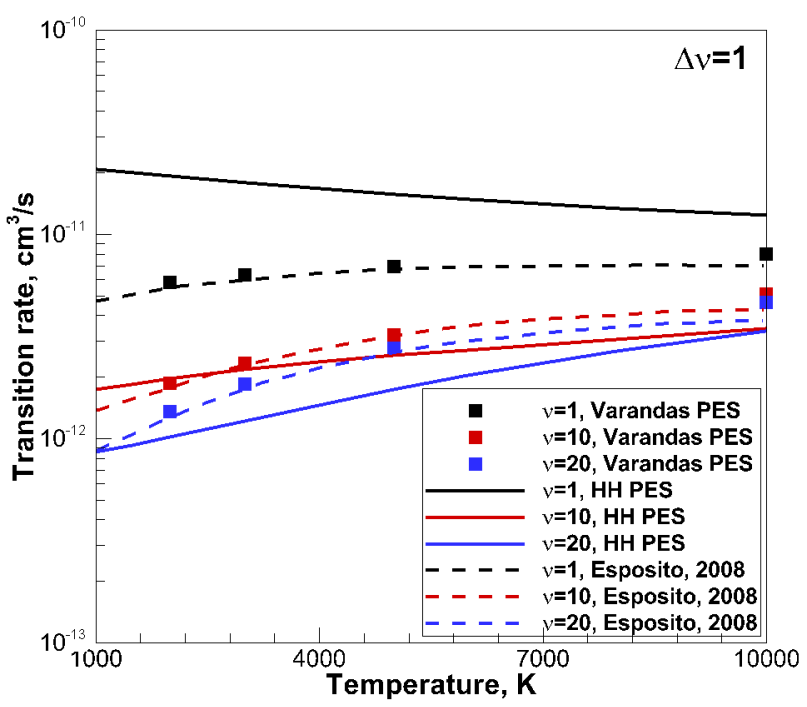

(a) $\Delta \nu=1, \nu=1,10$ and 20

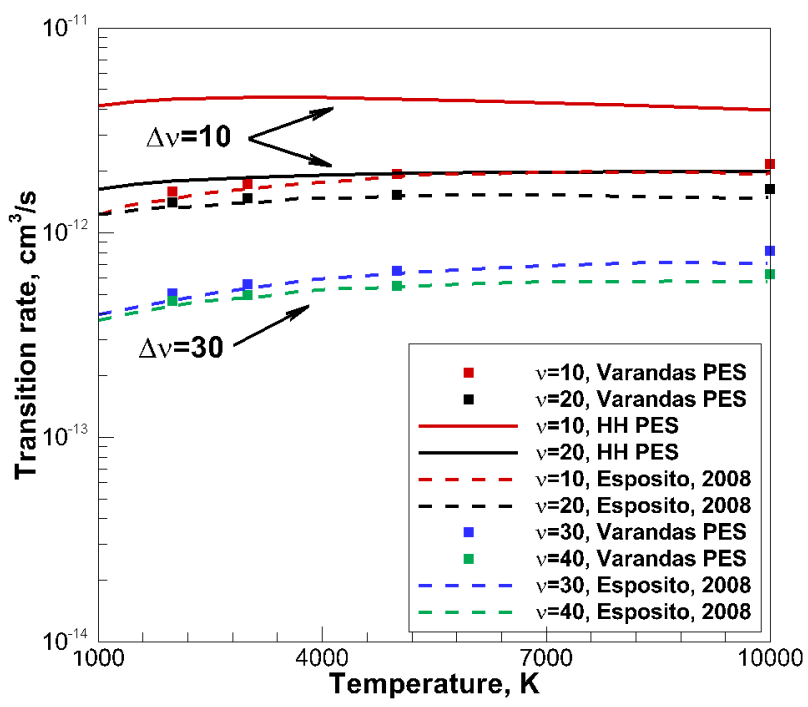

(b) $\Delta \nu=10$ and $30, \nu=10,20,30$ and 40

Fig. 2: Mono- and multiquantum deactivation rates

From Fig. 2 it follows that the variation of $\mathrm{O}_{2}-\mathrm{O}$ VT rates is relatively small over the wide temperature range. This is also true for the large multiquantum transitions. Meanwhile, the rate of monoquantum deactivation from $\nu_{i}=1$, shown in Fig. 2a by solid dashed lines, only slightly increases toward high temperature, suggesting that the relaxation time may also increase with the temperature. It is interesting to compare the present results to the transition rates observed in collisions of oxygen with an inert atom, such as argon [3]. The comparison of VT rates, generated by the QCT method, is shown in Figs. 3a and 3b for the $\mathrm{O}_{2}-\mathrm{O}$ and $\mathrm{O}_{2}-\mathrm{Ar}$ systems, respectively.

In both cases, the VT transition rates are calculated at translational temperatures of 2,000, 3,000, 5,000 and $10,000 \mathrm{~K}$. The lowest kinetic temperature corresponds to the lowest surface. Trans-rotational equilibrium is assumed. Rates on the left of the peak correspond to exothermic transitions. Rates of endothermic transitions, calculated using the principle of detailed balance, are shown on the right of the peak. One can see a very weak dependence of $\mathrm{O}_{2}-\mathrm{O}$ rates on temperature, while the efficiency of vibrational deactivation in $\mathrm{O}_{2}-\mathrm{Ar}$ collisions strongly depends on the translation temperature. Moreover, there is a small variation 
of $\mathrm{O}_{2}-\mathrm{O}$ exothermic rates with the initial vibrational quantum number. The present results demonstrate a small decrease of VT rates at very high vibrational levels due to the dissociation channel. On the other hand, $\mathrm{O}_{2}-\mathrm{Ar}$ vibrational deactivation rates strongly depend on $\nu_{i}$. Apparently, the difference in behavior of $\mathrm{O}_{2}-\mathrm{O}$ VT rates from the $\mathrm{O}_{2}-\mathrm{Ar}$ system is caused by a significant contribution of the repulsive component in the $\mathrm{O}_{2}-\mathrm{Ar}$ PES that is absent in the $\mathrm{O}_{3}$ PES.

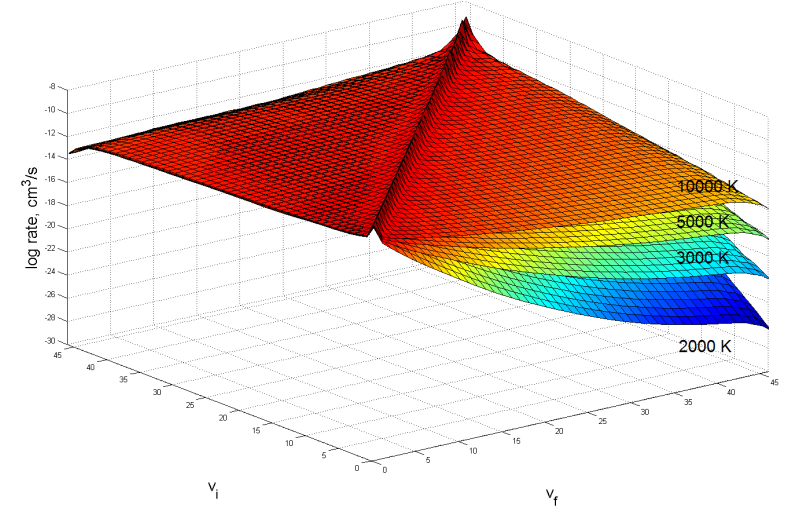

(a) $\mathrm{O}_{2}-\mathrm{O}$, Varandas PES

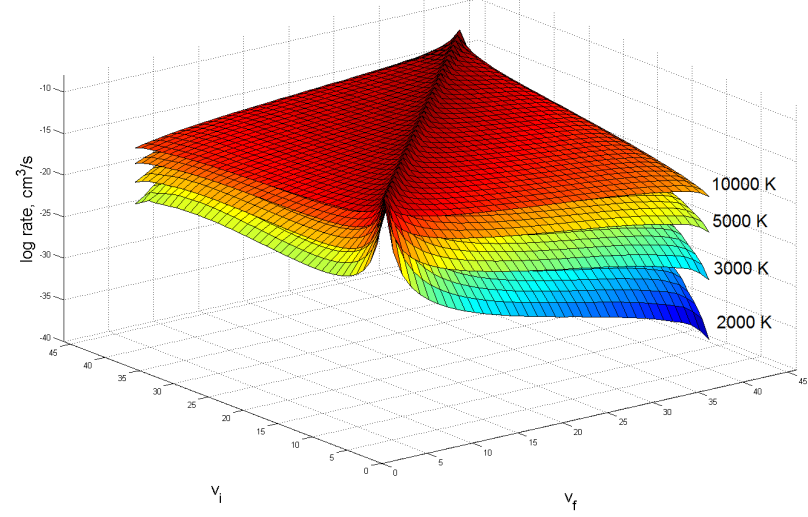

(b) $\mathrm{O}_{2}-\mathrm{Ar}[3]$

Fig. 3: VT transition rates of different atom-molecule systems

\section{B. VT and RVT models}

The solution of Eq. (12) describes a time-dependent population of each rovibrational level during the relaxation to thermal equilibrium. Because the large number of states complicates further analysis, the energy-equivalent rotational and vibrational temperatures are used to describe the population of the entire rovibrational ladder. The average vibrational and rotational energies are evaluated in the following manner:

$$
\begin{gathered}
\bar{e}_{\text {rot }}=\frac{\sum_{i} e_{\text {rot }, i} n_{i}}{\sum_{i} n_{i}} \\
\bar{e}_{v i b}=\frac{\sum_{i} e_{v i b, i} n_{i}}{\sum_{i} n_{i}},
\end{gathered}
$$

The rotational and vibrational temperatures are evaluated from $\bar{e}_{\text {rot }}$ and $\bar{e}_{v i b}$ by solving the following implicit equations:

$$
\begin{aligned}
& \bar{e}_{\text {rot }}=e_{\text {rot }}\left(T_{\text {rot }}, T_{v i b}\right), \\
& \bar{e}_{v i b}=e_{v i b}\left(T_{r o t}, T_{v i b}\right),
\end{aligned}
$$

where

$$
\begin{gathered}
e_{\text {rot }}\left(T_{r o t}, T_{v i b}\right)=\frac{1}{Q^{R V}} \sum_{\nu} \exp \left(-\frac{e_{v, 0}}{k_{B} T_{v i b}}\right) \sum_{j}(2 j+1)\left(e_{v, j}-e_{v, 0}\right) \exp \left(-\frac{\left(e_{v, j}-e_{v, 0}\right)}{k_{B} T_{v i b}}\right), \\
e_{v i b}\left(T_{r o t}, T_{v i b}\right)=\frac{1}{Q^{R V}} \sum_{\nu} e_{v, 0} \exp \left(-\frac{e_{v, 0}}{k_{B} T_{v i b}}\right) \sum_{j}(2 j+1) \exp \left(-\frac{\left(e_{v, j}-e_{v, 0}\right)}{k_{B} T_{v i b}}\right) .
\end{gathered}
$$

In Eqs. (19) and (20), $Q^{R V}$ is the total rovibrational partition function of oxygen:

$$
Q^{R V}=\sum_{\nu} \exp \left(-\frac{e_{\nu, 0}}{k_{B} T_{v i b}}\right) \sum_{j}(2 j+1) \exp \left(-\frac{e_{\nu, j}-e_{\nu, 0}}{k_{B} T_{r o t}}\right)
$$


The solution of Eqs. (17) and (18) is obtained by the bisection method. It is important to note that the vibrational and rotational temperatures, defined by Eqs. (17) - (21), are involved only in the post-processing step, after the solution of the master equations has been obtained. The evolution of vibrational temperature, computed under the heat bath conditions at $T=1,000 \mathrm{~K}, 5,000 \mathrm{~K}$ and $10,000 \mathrm{~K}$ using rates corresponding to the HH PES, is shown in Fig. 4a. Solid and dashed lines correspond to the RVT and VT models, respectively. For the entire range of translational temperature, the VT model predicts faster vibrational relaxation than the RVT model. This difference is caused by a significant rotational nonequilibrium in $\mathrm{O}_{2}-\mathrm{O}$ collisions. In fact, as will be shown later, the vibrational relaxation in collision of oxygen with the parent atom is only slightly faster than the rotational relaxation.

Comparison of VT models using two sets of reaction rates from the HH and Varandas potential energy surfaces is shown in Fig. 4b. As one can see, the solution of master equations using the Varandas PES predicts slower relaxation of vibrational energy than that by the HH PES. This result is expected from the general behavior of the VT rates in Fig. 2. It is worth to note that the difference in the vibrational relaxation time becomes smaller as translational temperature increases, since at these conditions colliding particles interact mostly via the repulsive part of the potential.

The evolution of vibrational and rotational temperatures given by the RVT model using rates from the HH PES is shown in Fig. 4c. In the range of heat bath temperature between 1,000 and 10,000 $\mathrm{K} \mathrm{a}$ significant degree of vibrational and rotational nonequilibrium is observed. The latter explains the difference in $T_{v i b}$ given by the VT and RVT models in Fig. 4a. Note, that at the early stage of relaxation, the rotational temperature is always lower than the vibrational temperature. This situation is also observed for $\mathrm{N}_{2}-\mathrm{N}$ collisions [2]. Eventually, the rotational temperature becomes greater than $T_{v i b}$ at the late stage of relaxation. During the QSS relaxation phase, when modeling dissociation and recombination, the rotational temperature is always larger than the vibrational temperature. This feature is left for further investigation.

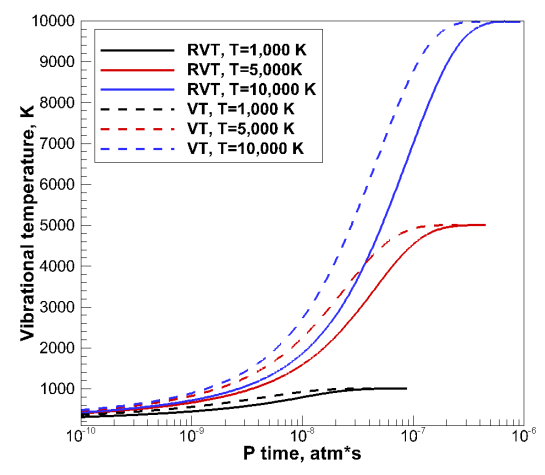

(a) HH PES, VT and RVT models

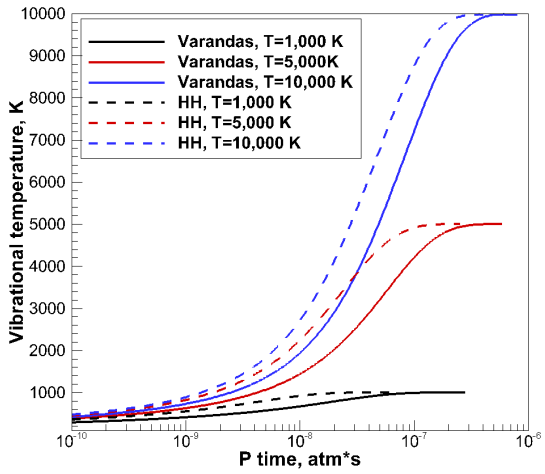

(b) HH and Varandas PESs, VT model

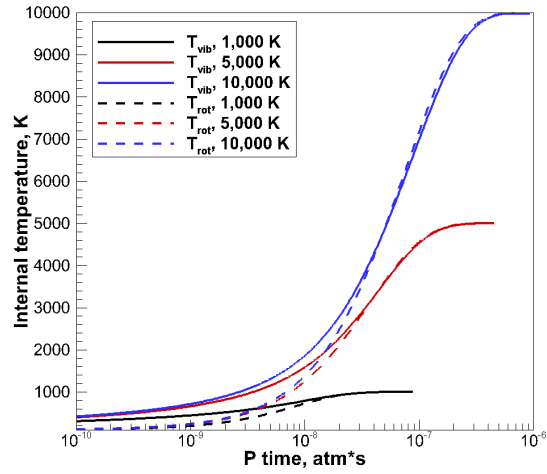

(c) HH PES, RVT model

Fig. 4: Evolution of vibrational and rotational temperatures

It is possible to define the vibrational and rotational relaxation times based on the temporal evolution of internal energy under the heat bath conditions. There are several ways to define a characteristic relaxation time [22]. One of the ways is to calculate the relaxation time as if the equilibration process follows the Landau-Teller equation:

$$
\frac{d e_{v}(t)}{d t}=\frac{e_{v i b}(t, T)-e_{v i b}\left(t, T_{v i b}\right)}{\tau_{v i b}} .
$$

The vibrational energy, given by Eq. (22), evolves exponentially in time. The vibrational relaxation time, $\tau_{v i b}$, is then defined by the corresponding vibrational energy $e_{v, \text { efold }}$ :

$$
e_{v i b, e f o l d}=\frac{1}{e} e_{v i b}\left(T_{0}\right)+\left(1-\frac{1}{e}\right) e_{v i b}(T) \approx 0.3679 \times e_{v i b}\left(T_{0}\right)+0.6321 \times e_{v i b}(T),
$$

where $e_{v i b, e f o l d}$ is the average vibrational energy at the time $\tau_{v i b}, e_{v i b}\left(T_{0}\right)$ and $e_{v i b}(T)$ are the average vibrational energies evaluated at initial and final temperatures, respectively. This approach is called the e-folding method [22]. The rotational relaxation time is calculated in a similar way. 
The present rotational and vibrational relaxation times are shown in Fig. 5. The black solid and dashed lines correspond to $\mathrm{P} \tau_{v i b}$ obtained by the VT model, using the Varandas and HH PESs, respectively. The black dashed-dotted line corresponds to the vibrational relaxation time obtained by the RVT model from the HH PES. The red dashed line presents $\mathrm{P} \tau_{\text {rot }}$ obtained from the RVT model based on the HH PES. Circular and diamond symbols represent the vibrational relaxation time measured in experiments by Breen at al. [12] and Kiefer and Lutz [13], respectively. The symboled line corresponds to the curve fit [9] of vibrational relaxation time using the Millikan-White relation to the data from [13].

The vibrational relaxation time obtained from the VT thermodynamic model based on the Varandas PES is in good agreement with the existing experimental data in the range from 1,000 $\mathrm{K}$ to $3600 \mathrm{~K}$. The best agreement is achieved with the data by Breen at al. The agreement with the earlier measurements by Kiefer and Lutz is less successful. The largest difference is observed in the high temperature region and is not higher than $15 \%$. Taking into account the overall uncertainty of the shock tube facility, the agreement with the experimental data is very satisfactory. To the authors' knowledge, other experimental data on the $\mathrm{O}_{2}-\mathrm{O}$ relaxation in this temperature range, as well as other simulations that adopt a master equation, do not exist.

Both the RVT and VT vibrational relaxation times based on the HH PES are smaller than the relaxation time from the Varandas PES. This result demonstrates the influence of the three-body interaction in the $\mathrm{O}_{2}-\mathrm{O}$ collisions. The agreement of the RVT HH vibrational relaxation time with the experimental data is very good, however one should keep in mind that no bimolecular collisions are introduced in the present simulation, thus, the actual RVT vibrational relaxation time in the $\mathrm{O}_{2}-\mathrm{O}$ mixture may be different.

The direct extrapolation of vibrational relaxation time by Kiefer and Lutz outside of the experimental range by utilizing the Millikan-White's relation (symboled curve) shows a significant difference from the present models based on either Varandas or HH potentials. The MW expression suggests that the vibrational relaxation of oxygen by the parent atom becomes more efficient with temperature [23]. The present results support a directly opposite trend: the vibrational relaxation time becomes smaller at low temperatures. This observation is in agreement with the results by Quack [5].

Another interesting feature of the present results is the strong coupling between the vibrational and rotational relaxation. The results by the RVT thermodynamic model suggest that the rotational relaxation is only slightly faster than the vibrational relaxation in the range of temperatures from 500 to $10,000 \mathrm{~K}$. The linking between $\tau_{v i b}$ and $\tau_{\text {rot }}$ explains the large difference in the vibrational temperature obtained by means of the VT and RVT models in Fig. 4a. The presence of rotational nonequilibrium at high temperatures is in the agreement with recent QCT simulations of other molecular systems [1,3], while at low temperatures the $\mathrm{O}_{2}-\mathrm{O}$ rotational nonequilibrium is explained by the anomalously fast vibrational relaxation.

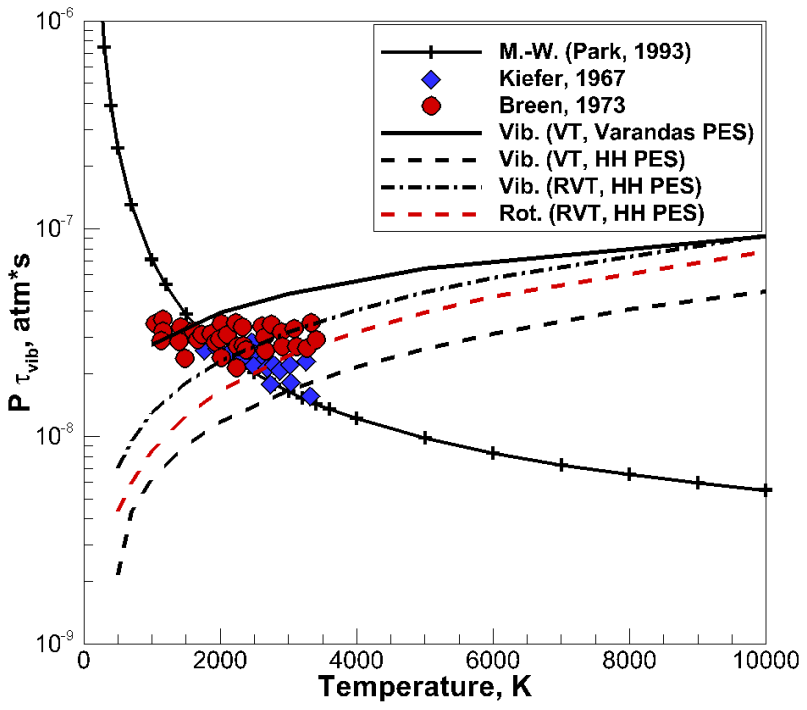

Fig. 5: Rotational and vibrational relaxation time

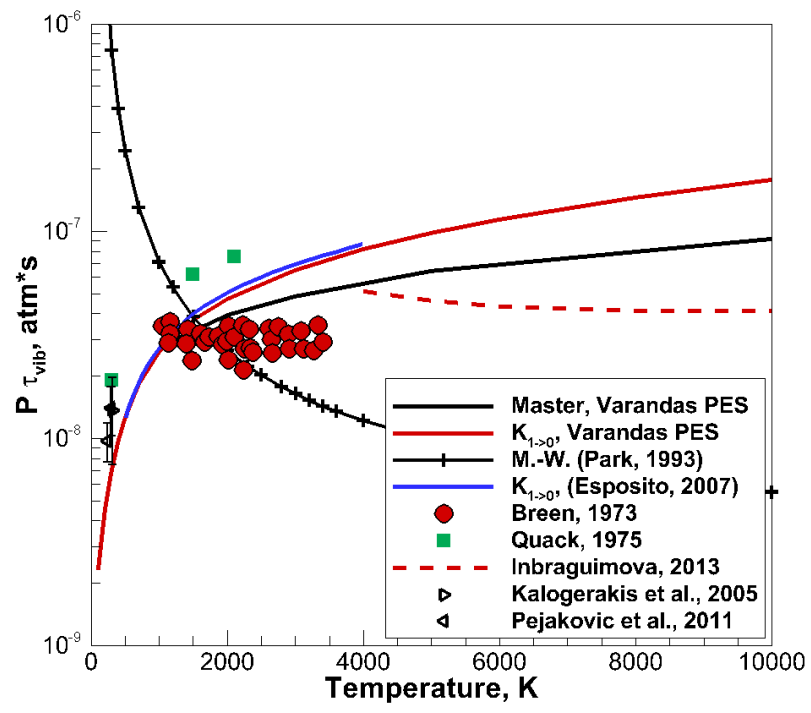

Fig. 6: Vibrational relaxation time

Results of the VT model using the Varandas PES are compared to the vibrational relaxation time derived from the rate of monoquantum deactivation and to that reported by Esposito et al. [24] and Ibraguimova 
et al. [25] in Fig. 6. Solid black and red curves describe the vibrational relaxation time by the VT model and the corresponding rate of monoquntum deactivation from $\nu_{i}=1$. Circular symbols correspond to the experimental data from [12]. The relaxation time, given by the dashed line, is taken from the experimental work [25]. The solid blue line represents the relaxation time derived from the rate of monoquantum deactivation by Esposito [4]. The symboled line and square symbols present Park's curve fit and the theoretical data by Quack and Troe [5].

Results by Ibraguimova et al. [25] were obtained by measuring the vibrational temperature behind shock waves in pure oxygen. The experimental data was then fitted to the general form of vibrational relaxation time [26]:

$$
P \tau_{v i b}=\frac{1}{P P_{10} \sigma_{0}} \sqrt{\frac{\pi \mu}{8 R T}} \frac{1}{1-\exp (-\theta / T)}, \text { Atm } \times s,
$$

where $P$ is the pressure, $P_{10}$ is the probability of vibrational deactivation, $\sigma_{0}$ is the collisional cross section, $\mu$ and $\theta$ are the reduced mass of colliding particles and characteristic vibrational temperature of the target molecule. The vibrational relaxation time, given by Eq. (24), asymptotically converges to the MW equation at low temperatures. At higher temperatures, Eq. (24) suggest slower vibrational relaxation due to the breakdown of the principles assumed in the Landau-Teller theory. Equation (24) was used in [25] to derive the $\mathrm{O}_{2}-\mathrm{O}_{2}$ and $\mathrm{O}_{2}-\mathrm{O}$ relaxation times. The relaxation times, reported in [25], provide a good description of vibrational temperature behind shock waves in the range of temperatures from $4000 \mathrm{~K}$ to $10800 \mathrm{~K}$. The agreement between results in [25] and those generated by the present VT model is very satisfactory and both results are substantially different from the MW relation. At the same time, the relaxation times calculated from the $\mathrm{K}_{1 \rightarrow 0}$ rate in the present work and in [24] are in the excellent agreement. This observation confirms the adequacy of the QCT method, employed in the present work.

The vibrational and rotational relaxation times calculated by the e-folding method can be fit by a polynomial function of translational temperature in the range from 1,000 to $20,000 \mathrm{~K}$. The following function is used to approximate the relaxation times:

$$
P \tau_{x}=10^{-8}\left[A\left(\frac{T}{1000}\right)^{3}+B\left(\frac{T}{1000}\right)^{2}+C\left(\frac{T}{1000}\right)+D\right],
$$

where $x$ stands for either vibrational or rotational mode, $P \tau_{x}$ is in Atm $\times \mathrm{s}, \mathrm{T}$ is in K. Coefficients A, B, C and $\mathrm{D}$ are given in Table 1 . The root mean square error of the curve fit is not larger than $1.5 \times 10^{-3} \%$.

\begin{tabular}{ccccc}
\hline \hline & $\mathrm{A}$ & $\mathrm{B}$ & $\mathrm{C}$ & $\mathrm{D}$ \\
\hline$\tau_{v i b}, \mathrm{RVT}, \mathrm{HH}$ PES & $-3.732 \times 10^{-4}$ & $-3.254 \times 10^{-3}$ & 0.949 & 0.3549 \\
$\tau_{r o t}, \mathrm{RVT}, \mathrm{HH}$ PES & $-4.399 \times 10^{-4}$ & $2.823 \times 10^{-3}$ & 0.776 & $7.223 \times 10^{-2}$ \\
$\tau_{v i b}, \mathrm{VT}, \mathrm{HH}$ PES & $-4.407 \times 10^{-6}$ & $-5.662 \times 10^{-3}$ & 0.5433 & $8.702 \times 10^{-2}$ \\
$\tau_{v i b}, \mathrm{VT}$, Varandas PES & $2.304 \times 10^{-3}$ & $-7.254 \times 10^{-2}$ & 1.245 & 1.7 \\
\hline \hline
\end{tabular}

Table 1: Curve fit parameters of relaxation times

The relaxation time, obtained by means of the RVT model, is used to verify the adequacy of the LandauTeller model, given by Eq. (22). The evolution of average vibrational and rotational energies, calculated from Eq. (22) using the relaxation parameters from Table 1 is compared to $\bar{e}_{v i b}$ and $\bar{e}_{\text {rot }}$ obtained from Eq. (16) and (15) in Figs. 7a and 7b. Chemical reactions are not considered. The pressure is set to $1.3625 \mathrm{Atm}$ at a heat bath temperature of 10,000 K. The Landau-Teller model perfectly describes the average energy of the rovibrational ladder at kinetic temperatures ranging from 1,000 to 20,000 K using the relaxation time derived in the present work.

In order to make the results applicable in CFD codes, the state-resolved $\mathrm{O}_{2}-\mathrm{O}$ VT transition rates based on the accurate Varandas PES are curve fitted in the range of translation temperature from 1,000 to 20,000 $\mathrm{K}$. In present work the following function is used to describe the rates of vibrational deactivation:

$$
K\left(T, \nu_{i} \rightarrow \nu_{f}\right)=10^{-12} \exp \left(a_{1}+\frac{a_{2}}{\log (T)}+a_{3} \log (T)\right),
$$




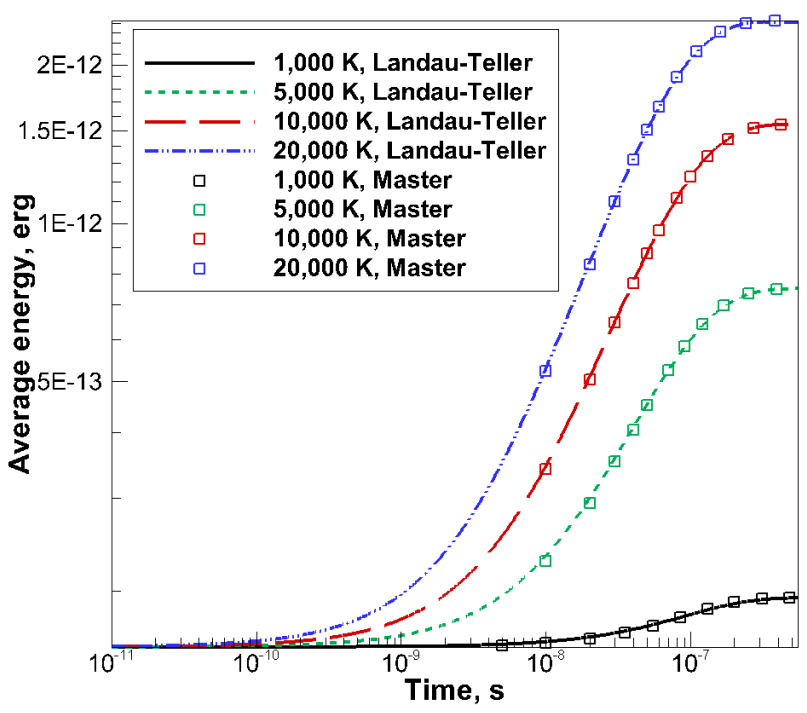

(a) Vibrational energy

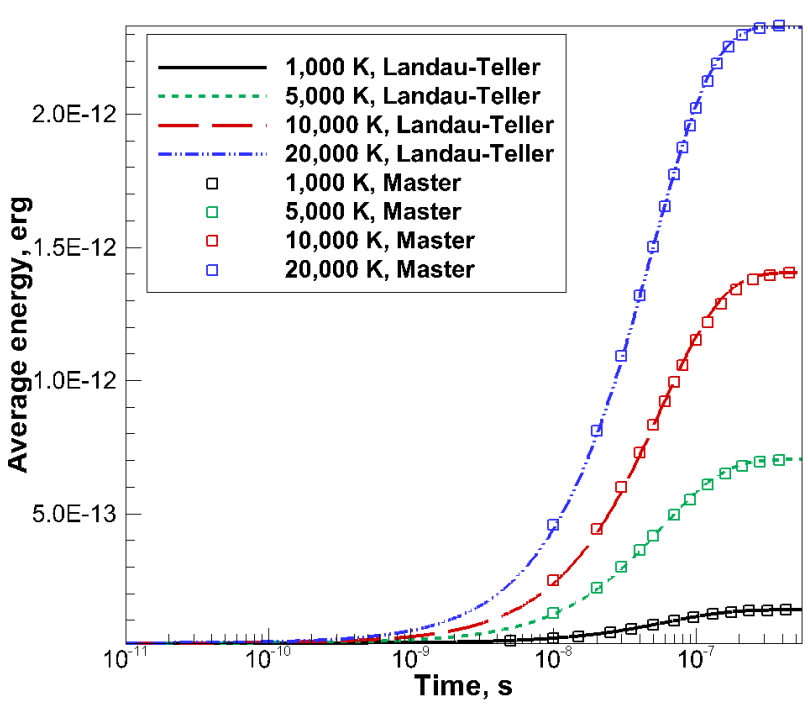

(b) Rotational energy

Fig. 7: Evolution of average internal energy

where $\nu_{i} \geq \nu_{f}, T$ is in $\mathrm{K}$, the transition rate, $K\left(T, \nu_{i} \rightarrow \nu_{f}\right)$, is in $\mathrm{cm}^{3} / \mathrm{s}$, and coefficients $a_{1}, a_{2}$ and $a_{3}$ for each of 1128 transitions are specified in Section VII. The present data has some important advantages over the results presented in [4]. The lower range of translational temperature is limited to 1,000 K; at lower temperatures the rates vary drastically while representing no interest to hypersonic simulations. Thus, the present curve fit coefficients have smaller error, compared to those in [4]. The maximum error is 15 $\%$ for $\nu=41-46$ and is less than $6 \%$ for the rest. Additionally, the present rates are more accurate at temperatures above $10,000 \mathrm{~K}$, since $\mathrm{O}_{2}-\mathrm{O}$ collisions are simulated up to the kinetic energy of $15 \mathrm{eV}$. The data, given in Table 2 , is available upon request.

\section{Analysis of RT and VT rates}

One can evaluate the rate of rotational-translational and vibrational-translational energy exchange using only the full set of rovibrational transition rates without involving the solution of master equations. The rate of total energy loss from vibrational level $\nu_{i}$ during the bound-bound transition to level $\nu_{f}$ can be expressed as follows [27]:

$$
R_{\nu_{i} \rightarrow \nu_{f}}=\frac{\sum_{j_{i}} e_{\nu_{i}, j_{i}}\left(2 j_{i}+1\right) \exp \left(-\left(e_{\nu_{i}, j_{i}}-e_{\nu_{i}, 0}\right) / k_{B} T_{r o t}\right) \sum_{j_{f}} K\left(T, \nu_{i}, j_{i} \rightarrow \nu_{f}, j_{f}\right)}{\sum_{j_{i}} e_{\nu_{i}, j_{i}}\left(2 j_{i}+1\right) \exp \left(-\left(e_{\nu_{i}, j_{i}}-e_{\nu_{i}, 0}\right) / k_{B} T_{r o t}\right)}
$$

It is assumed in Eq. (26) that the population of rotational levels is described by the Boltzmann distribution at temperature $T_{\text {rot }}$. The similar expression for the energy gain by vibrational level $\nu_{f}$ during the boundbound transition from the level $\nu_{i}$ can be written as follows:

$$
R_{\nu_{f} \leftarrow \nu_{i}}=\frac{\sum_{j_{i}}\left(2 j_{i}+1\right) \exp \left(-\left(e_{\nu_{i}, j_{i}}-e_{\nu_{i}, 0}\right) / k_{B} T_{r o t}\right) \sum_{j_{f}} e_{\nu_{f}, j_{f}} K\left(T, \nu_{i}, j_{i} \rightarrow \nu_{f}, j_{f}\right)}{\sum_{j_{i}} e_{\nu_{i}, j_{i}}\left(2 j_{i}+1\right) \exp \left(-\left(e_{\nu_{i}, j_{i}}-e_{\nu_{i}, 0}\right) / k_{B} T_{r o t}\right)}
$$

The loss and gain of energy, described by Eqs. (26) and (27), consist of vibrational and rotational contributions. To evaluate individual contributions, the vibrational energy of molecules in the ground rotational state must be subtracted:

$$
\begin{aligned}
& R_{\nu_{i} \rightarrow \nu_{f}}^{r o t}=R_{\nu_{i} \rightarrow \nu_{f}}-R_{\nu_{i} \rightarrow \nu_{f}}^{v i b}, \\
& R_{\nu_{f} \leftarrow \nu_{i}}^{r o t}=R_{\nu_{f} \leftarrow \nu_{i}}-R_{\nu_{f} \leftarrow \nu_{i}}^{v i b},
\end{aligned}
$$


where $R_{\nu_{i} \rightarrow \nu_{f}}^{v i b}=e_{v_{i}, 0} K\left(T, T_{r o t}, v_{i} \rightarrow v_{f}\right)$ and $R_{\nu_{f} \leftarrow \nu_{i}}^{v i b}=E_{v_{f}, 0} K\left(T, T_{r o t}, v_{f} \rightarrow v_{i}\right)$ are the loss and gain of vibrational energy by state $\nu_{i}$. The gain and loss of rotational energy due to the bound-bound transitions between $\nu_{i}$ and $\nu_{f}$ vibrational levels is given by Eqs. (28) and (29). The difference between $R_{\nu_{i} \rightarrow \nu_{f}}^{r o t}$ and $R_{\nu_{f} \leftarrow \nu_{i}}^{r o t}$ is due to the energy exchange between the rotational and translational degrees of freedom:

$$
R_{\nu_{i}, \nu_{f}}^{T, r o t}=R_{\nu_{i} \rightarrow \nu_{f}}^{r o t}-R_{\nu_{f} \leftarrow \nu_{i}}^{r o t}
$$

A similar exchange term can be derived for the trans-vibrational energy exchange:

$$
R_{\nu_{i}, \nu_{f}}^{T, v i b}=R_{\nu_{i} \rightarrow \nu_{f}}^{v i b}-R_{\nu_{f} \leftarrow \nu_{i}}^{v i b}
$$

Note, that $R_{\nu_{i} \rightarrow \nu_{f}}^{r o t}, R_{\nu_{i} \rightarrow \nu_{f}}^{v i b}, R_{\nu_{f} \leftarrow \nu_{i}}^{r o t}$ and $R_{\nu_{f} \leftarrow \nu_{i}}^{v i b}$ are positive, while $R_{\nu_{i}, \nu_{f}}^{T, r o t}$ and $R_{\nu_{i}, \nu_{f}}^{T, v i b}$ can be either positive or negative. Under shock conditions, when $T \gg T_{r}, T_{\nu}, R_{\nu_{i}, \nu_{f}}^{T, r o t}$ and $R_{\nu_{i}, \nu_{f}}^{T, v i b}$ are always positive, while in expanding flow they can be negative. Equations (26) - (30) allow an investigation of the internal energy exchange in a relatively simple manner.

It is interesting to compare the gain and loss of rovibrational energy in $\mathrm{O}_{2}-\mathrm{O}$ collisions to another atommolecule system. Colonna et al. [27] performed similar calculations for the collisions of the hydrogen molecule with its parent atom. The $\mathrm{O}_{2}-\mathrm{Ar}$ state-to-state rates also became available recently [3]. In the present work, a comparison of rotational and vibrational energy gain is performed for the fixed internal temperature $\mathrm{T}_{\text {rot }}$ $=\mathrm{T}_{v i b}=50 \mathrm{~K}$ and translational temperature from $10^{2}$ to $10^{5} \mathrm{~K}$. These parameters correspond to the typical conditions observed immediately behind a shock front.

$R^{T, r o t}$ and $R^{T, v i b}$ coefficients for the $\mathrm{O}_{2}-\mathrm{O}, \mathrm{H}_{2}-\mathrm{H}$ and $\mathrm{O}_{2}-\mathrm{Ar}$ systems are shown in Fig. 8. These results do not indicate which species proceeds to equilibrium faster than the others, since all rates are normalized to their own maximum values. The energy gain by the translational mode (not shown in Fig. 8) is always negative and asymptotically approaches unity for all translational temperatures. This represents the loss of energy by translational degree of freedom. The negative sign of $R^{r o t}$ and $R^{T, v i b}$ corresponds to the gain of energy. At low temperatures, nearly all energy from the translational mode is spent to excite the rotational degrees of freedom, while the vibrational mode remains virtually inactive. This regime is observed at temperatures below $8,000 \mathrm{~K}$ for $\mathrm{O}_{2}-\mathrm{Ar}, 1,000 \mathrm{~K}$ for hydrogen, and $400 \mathrm{~K}$ for $\mathrm{O}_{2}-\mathrm{O}$. As temperature increases further, the energy from translational motion is shared between the vibrational and rotational degrees of freedom. Note that the rate of energy gain by the rotational mode is always larger than that by the vibrational mode.

Recent master equation studies of state-to-state kinetics of hydrogen [28] revealed a significant transrotational nonequilibrium at kinetic temperatures of $6,000 \mathrm{~K}$ and higher. The presence of nonequilibrium can be judged by a relatively large deposition of kinetic energy into the vibrational degrees of freedom, compared to the rotational mode, as follows from Fig. 8. The same ratio of $R^{T, r o t}$ and $R^{T, v i b}$ for $\mathrm{O}_{2}-\mathrm{O}$ collisions is observed at temperatures as low as $2,000 \mathrm{~K}$. Comparing $R^{T, r o t}$ and $R^{T, v i b}$ for $\mathrm{O}_{2}-\mathrm{Ar}$ collisions, one may conclude, that the rotational nonequilibrium in this case occurs at higher temperatures than in $\mathrm{O}_{2}-\mathrm{O}$ and $\mathrm{H}_{2}-\mathrm{H}$ collisions. The solution of master equations, performed in [3], indicates that the rate of rotational relaxation becomes important at kinetic temperatures of $10,000 \mathrm{~K}$ and higher. This result is in agreement with the expectation of rotational nonequilibrium in $\mathrm{H}_{2}-\mathrm{H}$ and $\mathrm{O}_{2}-\mathrm{O}$ collisions.

It is interesting to compare the vibrational and rotational relaxation times in $\mathrm{O}_{2}-\mathrm{O}$ collisions with the relaxation times in pure oxygen as well as in $\mathrm{O}_{2}$ diluted by a chemically inactive gas, such as argon. An extensive overview of the experimental data on rotational relaxation in pure oxygen at temperatures below $1,500 \mathrm{~K}$ can be found in [29]. To the authors' knowledge, any experimental data on $\mathrm{O}_{2}$ rotational relaxation at higher temperatures does not exist. However, the rotational relaxation time in $\mathrm{O}_{2}-\mathrm{Ar}$ collisions was recently calculated by the QCT method over a wide temperature range [3]. The $\mathrm{O}_{2}-\mathrm{O}, \mathrm{O}_{2}-\mathrm{Ar}$ and $\mathrm{O}_{2}-$ $\mathrm{O}_{2}$ relaxation times are shown in Fig. 9. Diamond and delta symbols correspond to the vibrational and rotational relaxation times in pure oxygen, taken from [23] and [29]. Square symbols correspond to the $\mathrm{O}_{2}-\mathrm{Ar}$ vibrational relaxation time, measured in shock tube facilities [30]. Solid and short-dashed curves correspond to the vibrational and rotational relaxation times of $\mathrm{O}_{2}-\mathrm{O}$ obtained in the present work by means of the RVT model. Long-dashed and dashed-dotted lines describe the rotational and vibrational relaxation in $\mathrm{O}_{2}-\mathrm{Ar}[3]$.

It follows from Fig. 9 that the rotational nonequilibrium in $\mathrm{O}_{2}-\mathrm{Ar}$ collisions is significant only at temperatures of $10,000 \mathrm{~K}$ and higher. Below these temperatures, the rotational relaxation time is two to three orders 


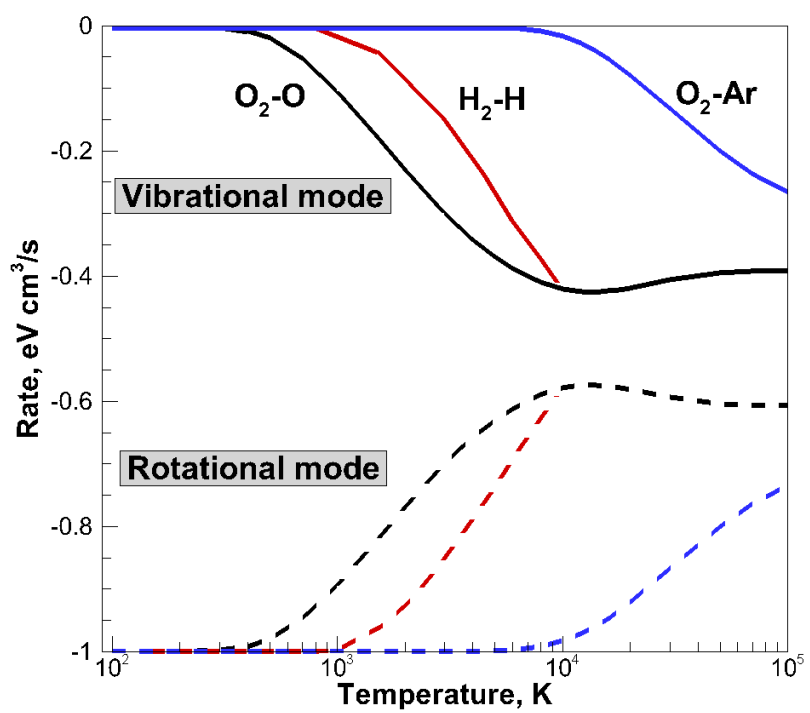

Fig. 8: Gain of energy by vibrational and rotational modes

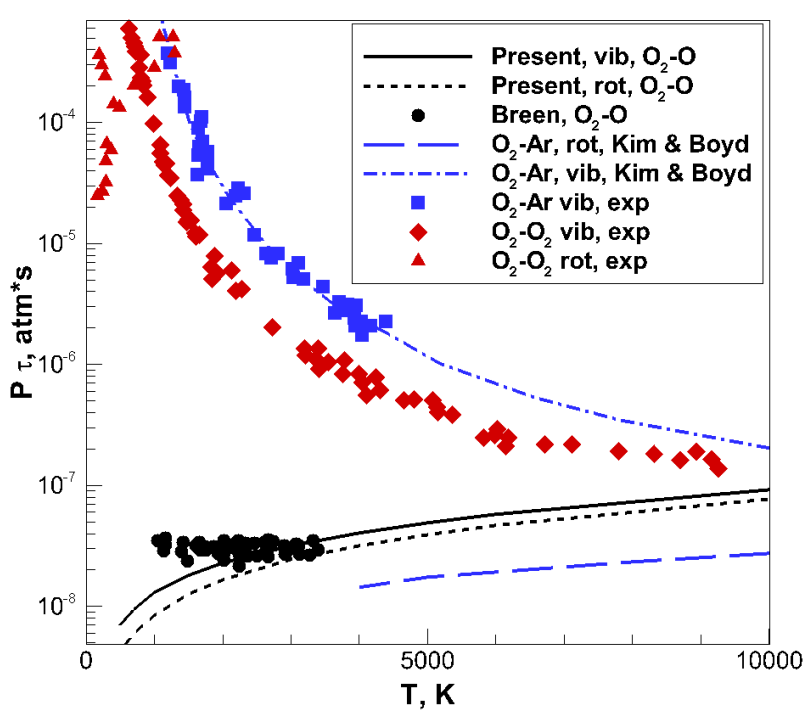

Fig. 9: Comparison of relaxation times in $\mathrm{O}_{2}-\mathrm{O}$, $\mathrm{O}_{2}-\mathrm{O}_{2}$ and $\mathrm{O}_{2}-\mathrm{Ar}$ collisions

of magnitude smaller than the vibrational relaxation time. This fact confirms the observation, concluded from Fig. 8. The $\mathrm{O}_{2}-\mathrm{O}_{2}$ and $\mathrm{O}_{2}-\mathrm{Ar}$ vibrational relaxation times are nearly the same order of magnitude for the entire temperature range, indicating that the mechanisms of trans-vibrational energy exchange in these systems are similar.

The experimental data on rotational relaxation in pure oxygen is ambiguous and is limited to temperatures from $150 \mathrm{~K}$ to $1,200 \mathrm{~K}$. The rotational relaxation in $\mathrm{O}_{2}-\mathrm{O}$ proceeds nearly three orders of magnitude faster that in pure oxygen. A similar conclusion can be made for the vibrational relaxation. More over, one can notice the opposite slopes of vibrational relaxation times for the $\mathrm{O}_{2}-\mathrm{O}_{2}, \mathrm{O}_{2}-\mathrm{Ar}$ and $\mathrm{O}_{2}-\mathrm{O}$ collisions. The explanation of this phenomenon lies in the different mechanisms of vibrational relaxation for the considered species.

\section{Nonreactive and exchange channels of reaction}

The exchange channel in collisions of oxygen with the parent atom has a significant impact on the process of internal energy transfer. Bauer and Tsang [31] have made an assumption that the vibrational relaxation in the $\mathrm{O}_{3}$ complex occurs entirely via the exchange channel. This observation was partially confirmed by Esposito and Capitelli [4]. They demonstrated the importance of the exchange channel by comparing rates of removal and monoquantum deactivation from the first excited vibrational level. The exchange mechanism contributes up to $30 \%$ to the total rate of monoquantum deactivation at temperatures below $300 \mathrm{~K}$, rapidly diminishing at high temperatures. Particular interest in the exchange reaction is due to its absence in collisions of oxygen with other species, such as argon. The present paper analyzes the contribution of the exchange reaction to the vibrational relaxation in a straightforward manner by computing the corresponding transition probability over a wide range of collision energies.

Vibrational transition probabilities via the exchange and nonreactive channels in $\mathrm{O}_{2}-\mathrm{O}$ and $\mathrm{O}_{2}-\mathrm{Ar}$ collisions are shown in Figs. 10a and 10b for the initial state $\left(\nu_{i}=1, j_{i}=1\right)$, respectively. Solid and dashed curves correspond to the exchange and nonreactive channels, respectively. The probability of vibrationally elastic transition is shown for reference by curves with symbols. One can see that vibrational transitions in $\mathrm{O}_{2}-\mathrm{O}$ collisions occur at much lower kinetic energies than in the $\mathrm{O}_{2}-\mathrm{Ar}$ interaction. In the latter case, the QCT method resolves only rotational transitions at collision energies lower than $1.7 \mathrm{eV}$. This fact explains an efficient rotational relaxation compared to the vibrational mode in the $\mathrm{O}_{2}-\mathrm{Ar}$ mixture. Here one should note that the number of trajectories, adopted for the $\mathrm{O}_{2}-\mathrm{Ar}$ QCT simulation was 2,000. The vibrational transition probability for these species at low temperatures is orders of magnitude smaller than that in $\mathrm{O}_{2}-\mathrm{O}$ collisions, as follows from Fig. 9. Thus, to obtain accurate vibrational transition probabilities for the $\mathrm{O}_{2}-\mathrm{Ar}$ system one should significantly increase the number of trajectories in the QCT simulation. At the same 
time, the probability of vibrational deactivation is comparable for the $\mathrm{O}_{2}-\mathrm{O}$ and $\mathrm{O}_{2}-\mathrm{Ar}$ systems at energies above $5 \mathrm{eV}$, which explains the close vibrational relaxation times in Fig. 5 .

The exchange and nonreactive channels of deactivation are both active in $\mathrm{O}_{2}-\mathrm{O}$ collisions starting from very low energy. The contribution of the exchange channel is significant for energies below $1 \mathrm{eV}$, however at higher energies, the exchange probability rapidly approaches to zero. The exchange probability during the vibrationally elastic collision is small for all considered energies.

The vibrational energy randomization is also studied for high lying levels in Figs. 11a and 11b for the initial state $v_{i}=20, j_{i}=1$. The probability of multi-quantum vibrational deactivation at low collision energies is strongly dependent on the exchange channel. The latter contribution is nearly twice as high as the probability of the nonreactive channel at kinetic energies between 0.1 and $1 \mathrm{eV}$. The contribution of the exchange channel diminishes at high energies, while the nonreactive channel remains open. This situation also takes place in transitions to higher vibrational states, as shown in Fig. 11b. More importantly, the exchange plays a dominant role in large multiquantum jumps rather than in transitions with small energy randomization, as follows from Fig. 11a. For the transition $\nu_{i}=20 \rightarrow \nu_{f}=10$, the nonreactive channel plays major role for energies of $1 \mathrm{eV}$ and higher, while for transition $\nu_{i}=20 \rightarrow \nu_{f}=0$, this takes place only at energies higher than $10 \mathrm{eV}$.

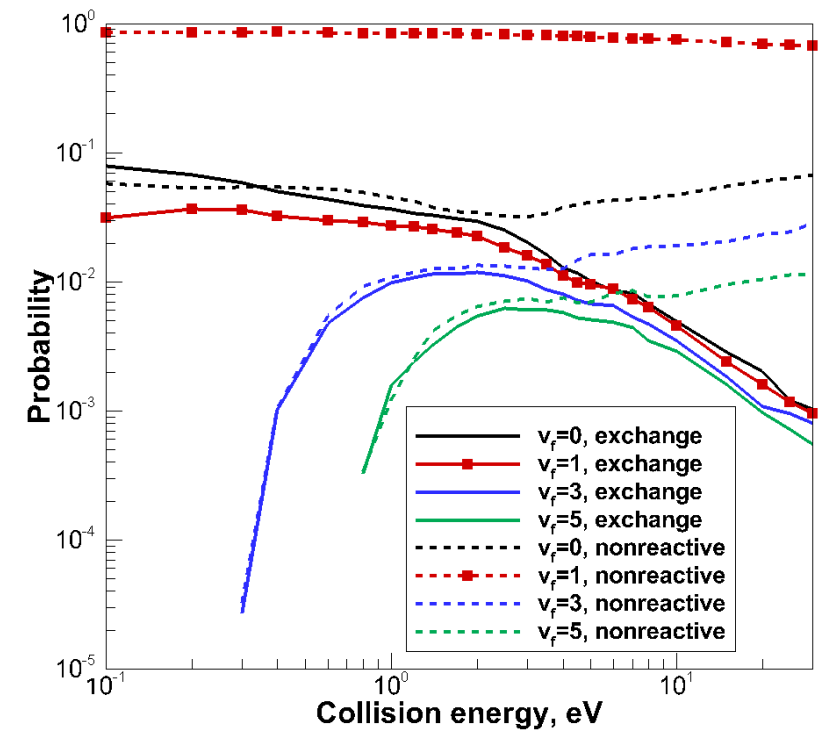

(a) $\mathrm{O}_{2}-\mathrm{O}$

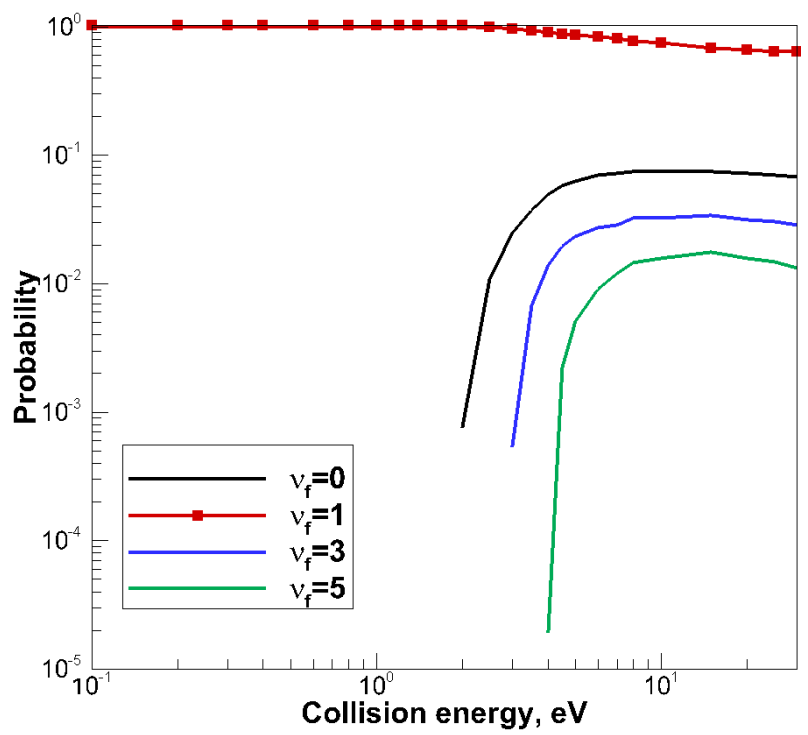

(b) $\mathrm{O}_{2}-\mathrm{Ar}$

Fig. 10: Transition probability for the state $\left(\nu_{i}=1, j_{i}=1\right)$

\section{Conclusion}

The vibrational and rotational relaxation of diatomic oxygen in collisions with its parent atom is studied by means of master equations, constructed for each vibrational and rovibrational state. The investigation is performed utilizing VT and RVT thermodynamic models in the range of heat bath conditions between 1,000 and 20,000 K. The state-specific rates are generated using two potential energy surfaces of different fidelity with 5,117 and 6,245 rovibrational states, respectively.

The present VT and RVT rates accurately describe the experimental data for $\mathrm{O}_{2}-\mathrm{O}$ vibrational relaxation. However, the relaxation time, derived by the e-folding method, has a significantly different temperature dependence compared to that by the conventional Millikan-White equation. Namely, the vibrational relaxation in $\mathrm{O}_{2}-\mathrm{O}$ collisions is very effective at low temperatures; the relaxation time slowly increases toward the high temperature limit. Meanwhile, it is postulated that the rates of vibrational deactivation weakly depend on the kinetic temperature. This situation leads to significant rotational nonequilibrium in the range of temperatures observed in hypersonic flows, as follows from the solution of the master equations.

The present work demonstrates that the utilization of an accurate potential energy surface is required for 


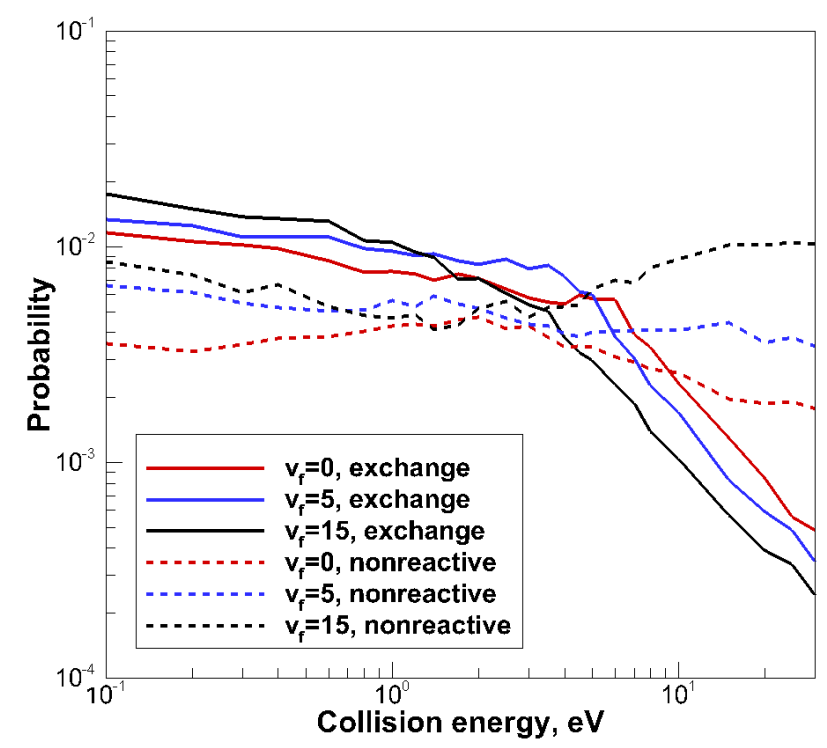

(a) Vibrational deactivation

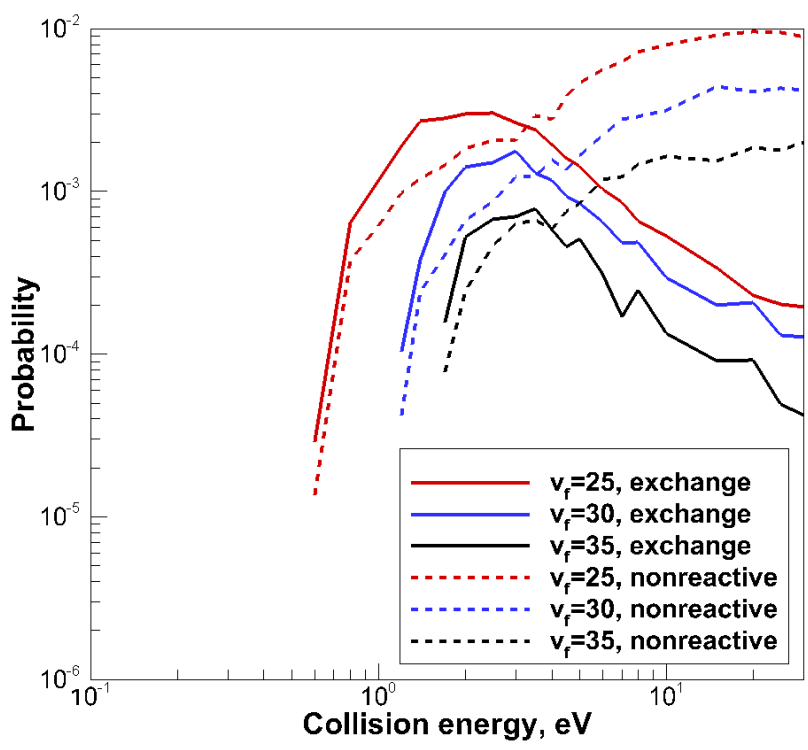

(b) Vibrational activation

Fig. 11: Vibrational transition probability in $\mathrm{O}_{2}-\mathrm{O}$ collisions for the state $\left(\nu_{i}=20, j_{i}=1\right)$

the adequate description of experimental data on $\mathrm{O}_{2}-\mathrm{O}$ relaxation. Failure to account for the many-body interaction in a chemically reactive system, such as $\mathrm{O}_{3}$, leads to the overestimation of vibrational deactivation rates. However, the difference in results between simple and accurate potential energy surfaces diminishes at high temperature. This is due to the fact that particles interact mostly via the repulsive part of the potential at these conditions.

In the present work, convenient relations for the averaged rotational and vibrational relaxation times are derived. It is demonstrated that the Landau-Teller model accurately describes the evolution of the average vibrational and rotational energies using the present relaxation parameters. The detailed analysis of stateto-state transition rates indicates that the rotational nonequilibrium in the $\mathrm{O}_{2}$ collisions with atomic oxygen takes place at much lower temperatures than in $\mathrm{H}_{2}-\mathrm{H}$ and $\mathrm{O}_{2}-\mathrm{Ar}$ mixtures. This fact may be important when modeling shock flows with a significant amount of oxygen atoms. Finally, a complete database of curve fitted VT transition rates, obtained for the accurate potential energy surface, is given in the Appendix.

\section{Acknowledgment}

The authors gratefully acknowledge funding for this work through Air Force Office of Scientific Research Grant FA9550-12-1-0483. DA would like to thank Dr. Jae Gang Kim for the $\mathrm{O}_{2}-$ Ar QCT program code and numerous fruitful discussions and Kevin Neitzel for the curve fitting tool. 
VII. Appendix

\begin{tabular}{|c|c|c|c|c|c|c|c|c|c|c|c|c|c|c|}
\hline$\nu_{i}$ & $\nu_{f}$ & a1 & $\mathrm{a} 2$ & a3 & $\nu_{i}$ & $\nu_{f}$ & a1 & $\mathrm{a} 2$ & a3 & $\nu_{i}$ & $\nu_{f}$ & a1 & $\mathrm{a} 2$ & a3 \\
\hline 1 & 1 & $.141-1$ & $22+0$ & $3.968-1$ & $y$ & 3 & $3.729+0$ & $-1.959+1$ & $-8.213-2$ & 15 & 0 & $.814+0$ & $-3.351+1$ & $-1.885-1$ \\
\hline 1 & 0 & $1.471-1$ & $-7.504-1$ & 2.214-1 & 11 & 8 & $-2.963+0$ & $6.811+0$ & $3.418-1$ & 16 & 16 & 7.934-1 & & \\
\hline 2 & 2 & $1.248+0$ & $-2.005-1$ & $3.563-1$ & 11 & 7 & 4.523-1 & $-6.499+0$ & $1.200-1$ & 16 & 15 & $-1.380+1$ & $3.859+1$ & 1.20 \\
\hline 2 & 1 & $-3.765+0$ & $1.029+1$ & 4.990-1 & 11 & 6 & $3.540+0$ & $-1.917+1$ & $-7.100-2$ & 16 & 14 & $9+1$ & $6.093+1$ & $1.260+0$ \\
\hline 2 & 0 & $4.723+0$ & $-1.883+1$ & $-1.070-1$ & 11 & 5 & $3.533+0$ & $-1.908+1$ & $-7.548-2$ & 16 & 13 & & $34+1$ & $8.260-1$ \\
\hline & & & & $3.785-1$ & 11 & 4 & $3+0$ & $19+1$ & $-1.648-1$ & 16 & 12 & $-7.528+0$ & $66+1$ & $5.757-1$ \\
\hline $\begin{array}{l}3 \\
3 \\
\end{array}$ & 2 & $-5.033+0$ & & & 11 & 3 & $9+0$ & $02+1$ & $733-1$ & 16 & 11 & $-3.697+0$ & $11+1$ & $3.278-1$ \\
\hline 3 & 0 & $\begin{array}{l}2.402+0 \\
5858+0\end{array}$ & $\begin{array}{l}-1.144+1 \\
-2.473+1\end{array}$ & $\begin{array}{r}4.142-2 \\
-1.846-1\end{array}$ & & 2 & $4.964+0$ & $-2.540+1$ & $-1.622-1$ & 16 & 10 & $-1.423+0$ & $4.228+0$ & 1.724-1 \\
\hline & & $3.073-1$ & $3.551+0$ & 4.079-1 & 11 & $\begin{array}{l}1 \\
0\end{array}$ & $4.752+0$ & $-2.479+1$ & $-1.503-1$ & $\begin{array}{l}10 \\
16\end{array}$ & S & $-3.608-1$ & $\begin{array}{l}.002+0 \\
3.969-1\end{array}$ & $9.794-2$ \\
\hline 4 & S & $-6.546+0$ & $1.593+1$ & 7.174-1 & 12 & 12 & $1.321+0$ & $-1.763+0$ & 3.481-1 & 16 & 7 & $2.894-1$ & $-3.795+0$ & $7.499-2$ \\
\hline 4 & ${ }_{1}^{2}$ & $\begin{array}{r}4.456-1 \\
4.552+0\end{array}$ & $\begin{array}{l}-5.513+0 \\
-2.006+1\end{array}$ & $\begin{array}{l}1.18-1 \\
-1.115-1\end{array}$ & 12 & $\begin{array}{l}11 \\
10\end{array}$ & $\begin{array}{l}-1.118+1 \\
-8.839+0\end{array}$ & $\begin{array}{l}2.844+1 \\
2706+1\end{array}$ & $\begin{array}{l}1.048+0 \\
7537-1\end{array}$ & $\begin{array}{l}16 \\
16\end{array}$ & 6 & $\begin{array}{l}-5.108-1 \\
-5.596-1\end{array}$ & $\begin{array}{l}-1.645+0 \\
-2501+0\end{array}$ & $\begin{array}{l}1.331-1 \\
1.455-1\end{array}$ \\
\hline 4 & 0 & $6.926+0$ & $-3.023+1$ & $-2.528-1$ & 12 & 9 & $-5.148+0$ & $1.616+1$ & $4.641-1$ & 16 & & $-3.790-$ & 5.000 & $\begin{array}{l}1.397-1 \\
1.397\end{array}$ \\
\hline & 5 & $2.040+0$ & $-3.822+0$ & 3.053-1 & 12 & 8 & $-8.920-1$ & $-2.272-2$ & $1.856-1$ & 16 & 3 & $8.470-$ & $13+0$ & $.265-2$ \\
\hline & 4 & $-6.987+0$ & $1.610+1$ & 7.584-1 & 12 & 7 & $2.666+0$ & $-1.498+1$ & $-2.901-2$ & 16 & 2 & $2.507+0$ & $-1.772+1$ & $-1.478-2$ \\
\hline 5 & 3 & $-1.481+0$ & $4.622-1$ & 3. $017-1$ & 12 & 6 & $4.304+0$ & $-2.210+1$ & $-1.257-1$ & 16 & 1 & $3.241+0$ & $-2.210+1$ & $-4.600-2$ \\
\hline$\frac{5}{5}$ & 2 & $\begin{array}{l}3.359+0 \\
5.830+0\end{array}$ & $-1.654+1$ & $\begin{array}{l}-3.504-2 \\
-1984-1\end{array}$ & 12 & 5 & $4.395+0$ & $-2.267+1$ & $-1.323-1$ & 16 & 0 & $5.090+0$ & $-3.056+1$ & $-1.499-1$ \\
\hline 5 & 0 & $7.029+0$ & $-3.134+1$ & $-2.644-1$ & 12 & 3 & $4.812+0$ & $\begin{array}{l}-2.0 .41+1 \\
-2.483+1\end{array}$ & $-1.570-1$ & 17 & $\begin{array}{l}11 \\
16\end{array}$ & $-1.261+1$ & $3.266+1$ & $\begin{array}{l}4.010-1 \\
1.147+0\end{array}$ \\
\hline$\rho$ & 6 & $1.606-1$ & $3.858+0$ & $4.175-1$ & 12 & 2 & $4.151+0$ & $-2.224+1$ & $-1.182-1$ & 17 & 15 & $-1.503+1$ & $5.056+1$ & $1.135+0$ \\
\hline 6 & 5 & -8.5 & $2.148+1$ & 8. & 12 & 1 & $.326+0$ & -2 & $-1.804-1$ & 17 & 14 & 1250 & $4170+1$ & $9.089-1$ \\
\hline 6 & 4 & $-3.085+0$ & $5.989+0$ & 4.036-1 & 12 & 0 & $5.248+0$ & $-2.822+1$ & $-1.707-1$ & 17 & 13 & $-8.699+0$ & & $6.427-1$ \\
\hline 6 & $\begin{array}{l}3 \\
2 \\
2\end{array}$ & $\begin{array}{l}1.376+0 \\
4\end{array}$ & $-9.496+0$ & 8.721-2 & 13 & 13 & $1.005+0$ & $-3.411-1$ & $3.637-1$ & 17 & 12 & $-4.481+0$ & +1 & 3.709-1 \\
\hline 6 & 2 & $\begin{array}{l}4.241+0 \\
5006+0\end{array}$ & $\begin{array}{l}-2.008+1 \\
-2.296+1\end{array}$ & $\begin{array}{l}-1.038-1 \\
-1.551-1\end{array}$ & 13 & 12 & $\begin{array}{l}-1.093+1 \\
-1030+1\end{array}$ & $2.695+1$ & $1.038+0$ & 17 & $\begin{array}{l}11 \\
10\end{array}$ & $-2.260+0$ & +0 & $2.269-1$ \\
\hline 6 & 0 & $7.354+0$ & $-3.297+1$ & $-2.919-1$ & 13 & 10 & $-5.100+0$ & $.517+1$ & 4.667-1 & 17 & 9 & $-1.394+0$ & $5.412+0$ & $1.490-1$ \\
\hline & 7 & $-3.333+0$ & $1.705+1$ & $6.440-1$ & 13 & 9 & $-1.740+0$ & $3.945+0$ & 2.270 & 17 & 8 & $4.067-3$ & -1. & $7.441-2$ \\
\hline 7 & 6 & $-8.792+0$ & $2.168+1$ & $8.806-1$ & 13 & 8 & $1.644+0$ & $-1.006+1$ & 2.146-2 & 17 & & $9.268-1$ & $-6.960+0$ & 3.885-2 \\
\hline 7 & 5 & $-4.002+0$ & 8.5 & & 13 & 7 & $4.847+0$ & & $-1.674-1$ & 17 & & 7.361-2 & $-4.568+0$ & 9.981-2 \\
\hline & $\begin{array}{l}4 \\
3\end{array}$ & $\begin{array}{l}-1.188-1 \\
3.090+0\end{array}$ & $\begin{array}{l}-4.374+0 \\
-1.630+1\end{array}$ & $\begin{array}{r}1.815-1 \\
-3.210-2\end{array}$ & $\begin{array}{l}13 \\
13\end{array}$ & $\begin{array}{l}0 \\
5\end{array}$ & $\begin{array}{l}4.474+0 \\
4.277+0\end{array}$ & $-2.195+1$ & $\begin{array}{l}-1.453-1 \\
-1.334-1\end{array}$ & $\begin{array}{l}17 \\
17\end{array}$ & 4 & $\begin{array}{l}4.410-1 \\
7.658-1\end{array}$ & $\begin{array}{l}-.033+0 \\
-9.197+0\end{array}$ & $\begin{array}{l}8.555-2 \\
7.395-2\end{array}$ \\
\hline 7 & 2 & 4.3 & -2.10 & $-1.181-1$ & 13 & 4 & $3.584+0$ & -1.947 & $-9.070-2$ & 17 & 3 & $1.611+0$ & -1.3 & $3.054-2$ \\
\hline 7 & 1 & $5.711+0$ & $-2.633+1$ & $-2.027-1$ & 13 & 3 & $4.206+0$ & -2.2 & $-1.255-1$ & 17 & 2 & $2.124+0$ & -1.8 & $4.988-3$ \\
\hline 8 & $\begin{array}{l}0 \\
8\end{array}$ & $\begin{array}{r}7.121+0 \\
-2679+1\end{array}$ & $\begin{array}{r}-3.208+1 \\
1.080+2\end{array}$ & $\begin{array}{l}-2.868-1 \\
2 \\
142+0\end{array}$ & 13 & 2 & $\begin{array}{l}4.665+0 \\
5.241+0\end{array}$ & $-2.524+1$ & $-1.438-1$ & 17 & 1 & $\begin{array}{l}2.508+0 \\
3.423+0\end{array}$ & $\begin{array}{l}-1.918+1 \\
-2.378+1\end{array}$ & $\begin{array}{l}-4.826-3 \\
-5\end{array}$ \\
\hline 8 & 7 & $-1.029+1$ & $2.745+1$ & $9.725-1$ & 13 & 0 & $5.515+0$ & $-3.092+1$ & $-1.739-1$ & 18 & 18 & 1.623-1 & & $4.132-1$ \\
\hline 8 & 6 & $-4.672+0$ & 1.0 & 5.11 & 14 & 14 & 2.1 & -4.8 & $2.963-$ & 18 & 17 & $-1.197+1$ & -1 & $1.112+0$ \\
\hline 8 & & $-6.407-1$ & -3.0 & 2.1 & 14 & 13 & $-1.114+1$ & $2.747+1$ & $1.052+0$ & 18 & 16 & 22 & & $1.157+0$ \\
\hline & & 1.5 & -1.0 & & 14 & 12 & -1.0 & & & 18 & 15 & & & $8.792-1$ \\
\hline 8 & 3 & $4.056+0$ & -2.04 & -9.8 & 14 & 11 & $-4.856+0$ & 1.307 & 4.61 & 18 & 14 & $-8.286+0$ & +1 & $6.218-1$ \\
\hline 8 & 2 & $\begin{array}{l}5.158+0 \\
5.544+0\end{array}$ & $-2.614+1$ & $\begin{array}{l}-1.0 \\
-1.9\end{array}$ & $\begin{array}{l}14 \\
14\end{array}$ & 90 & $\begin{array}{r}-1.900+0 \\
6.758-1\end{array}$ & $\begin{array}{l}4.0 \\
-5.7\end{array}$ & 7.46 & $\begin{array}{l}18 \\
18\end{array}$ & $\begin{array}{l}13 \\
12\end{array}$ & $\begin{array}{l}-2.727+0 \\
-2.727+0\end{array}$ & & $\begin{array}{l}3.984-1 \\
2.509-1\end{array}$ \\
\hline 8 & 0 & $6.833+0$ & $-3.110+1$ & $-2.768-1$ & 14 & 8 & $2.944+0$ & $-1.507+1$ & $-6.656-2$ & 18 & 11 & $-1.049+0$ & & $376-1$ \\
\hline 9 & 9 & 8 & 6.890-1 & 3.730-1 & 14 & 7 & $5.259+0$ & -2.5 & $-1.994-1$ & 18 & 10 & $-2.085-2$ & -6 & $6.977-2$ \\
\hline & 8 & $-1.129+1$ & $3.157+1$ & $1.030+0$ & 14 & 6 & $4.326+0$ & $-2.168+1$ & $-1.449-1$ & 18 & 9 & $5.626-1$ & & $9.650-2$ \\
\hline 9 & 7 & $-5.791+0$ & 1.5 & & 14 & 5 & $3.028+0$ & -1. & $-5.945-2$ & 18 & 8 & -2.1 & & $9.053-2$ \\
\hline 9 & 5 & $1.343+0$ & $-1.031+1$ & 7. $494-1$ & 14 & $\begin{array}{l}4 \\
3\end{array}$ & $3.341+0$ & $\begin{array}{l}-1.000 \\
-1.943\end{array}$ & $\begin{array}{l}-4.510-2 \\
-7.236-2\end{array}$ & 18 & 6 & $1.158+0$ & & $\begin{array}{l}8.095-2 \\
3650_{-}\end{array}$ \\
\hline 0 & 1 & & -1.8 & -2 & 14 & 2 & 5.2 & & & & 5 & $9.747-2$ & & $0.005-2$ \\
\hline 0 & & & -2.5 & & 14 & 1 & & & -1. & & 4 & & & 2 \\
\hline 0 & & 5.50 & -2.6 & -1.9 & 14 & 0 & 6.47 & & -2 & 18 & 3 & $1.219+0$ & & \\
\hline 0 & 0 & $5.478+0$ & -2.5 ? & $-1.992-1$ & 15 & 15 & 5.74 & 1.27 & 3.883-1 & 18 & 2 & & & \\
\hline 10 & 10 & 1.6 & -2.71 & 3.2 & 15 & 14 & 10 & & $1.110+0$ & 1 & 1 & $9-1$ & & \\
\hline 10 & 9 & $-1.230+1$ & $3.529+1$ & $1.093+0$ & 15 & 13 & -1.18 & & $94 \mathrm{C}$ & 18 & 0 & $2931+0$ & & $\begin{array}{r}9.1 \\
-3.3 \\
\end{array}$ \\
\hline & 8 & $-7.232+0$ & $2.131+1$ & $6.547-1$ & 15 & 12 & $-7.316+0$ & 2.290 & $6084-1$ & 19 & 19 & $5-2$ & & $\begin{array}{r}-3.350-2 \\
4.250-1\end{array}$ \\
\hline 10 & 7 & -1.53 & $2.578-1$ & & 15 & 11 & $-3.364+0$ & $8.979+0$ & $3.365-1$ & 19 & 18 & $-1.102+1$ & & $1.058+0$ \\
\hline 10 & 6 & 1.0 & -9.0 & 9.2 & 15 & 10 & -7.7 & & & & 17 & & & $1.193+0$ \\
\hline 10 & 5 & & -1.4 & & I0 & 9 & & & & 19 & 16 & & & $9.253-1$ \\
\hline 10 & 1 & 4.0 & -2.1 & & 15 & 8 & 3.16 & & & 19 & 15 & & & \\
\hline 10 & 3 & 4.6 & -2.3 & -1.4 & 15 & 7 & 3.5 & & $-1 .($ & 19 & 14 & & & $4.217-1$ \\
\hline 10 & 2 & 4.7 & -2.4 & -1. & 15 & 6 & & & & 19 & 13 & -3.5 & & $2.986-1$ \\
\hline 10 & 1 & $5.097+0$ & $-2.530+1$ & -1.7 & 15 & 5 & & & & 19 & 12 & -1.9 & & 1.87 \\
\hline 10 & 0 & $5.081+0$ & $-2.517+1$ & $-1.727-1$ & 15 & 4 & $2.722+0$ & $-1.696+1$ & $-3.670-2$ & 19 & 11 & $-1.100+0$ & & $1.330-1$ \\
\hline 11 & 11 & $8.000-1$ & $6.424-1$ & $3.774-1$ & 15 & 3 & $3.361+0$ & $-1.990+1$ & -7.4 & 19 & 10 & -1.18 & & $99-1$ \\
\hline 11 & 10 & -1.18 & $3.248+1$ & $1.080+0$ & 15 & 2 & $3.832+0$ & & -9.12 & & 9 & & & \\
\hline & & $-87 f<-$ & $2.769+1$ & 7.420-1 & 15 & 1 & $5.241+0$ & -3.0 & $-1.590-1$ & 19 & 8 & $4-1$ & -4.9 & $4.699-2$ \\
\hline
\end{tabular}

Table 2: Curve fit coefficients of VT transitions rates 


\begin{tabular}{|c|c|c|c|c|c|c|c|c|c|c|c|c|c|}
\hline$\nu_{f}$ & a1 & $\mathrm{a} 2$ & a3 & $\nu_{i}$ & $\nu_{f}$ & a1 & $\mathrm{a} 2$ & a.3 & $\nu_{i}$ & $\nu_{f}$ & a1 & $\mathrm{a} 2$ & a.3 \\
\hline 19 & $743+0$ & -1.131 & $-6.184-3$ & 22 & 6 & $1.062+0$ & -1.050 & $4.368-2$ & 25 & 14 & $-8.841-1$ & $1.044+0$ & $1.175-1$ \\
\hline 19 & $1.166+0$ & $-1.051+1$ & $4.437-2$ & 22 & 5 & 8.395-1 & -1.022 & $6.094-2$ & 25 & 13 & 4.113-1 & $-4.253+0$ & $4.046-2$ \\
\hline 19 & 7.955-1 & $-9.422+0$ & $6.720-2$ & $\overline{2} \overline{2}$ & 4 & $-1.250+0$ & $-2.347+0$ & $1.908-1$ & 25 & 12 & $-7.312-1$ & $8.405-1$ & 1.058-1 \\
\hline 19 & $9.143-1$ & $-1.049+1$ & $6.383-\overline{2}$ & $\overline{2} \overline{2}$ & 3 & $-6.680-1$ & $-5.272+0$ & $1.582-1$ & 25 & 11 & $6.765-2$ & $-2.305+0$ & $5.576-2$ \\
\hline 19 & 4.881-1 & $-9.459+0$ & $9.590-\overline{2}$ & $\overline{2} \overline{2}$ & 2 & $-1.652+0$ & -1.272 & $2.134-1$ & 25 & 10 & $1.911-1$ & $-2.939+0$ & $4.931-2$ \\
\hline 19 & $-6.616-2$ & $-7.624+0$ & $1.302-1$ & $\overline{2} \overline{2}$ & $\overline{1}$ & $-1.207+0$ & -3.391 & $1.863-1$ & 25 & 9 & $6.425-1$ & $-5.377+0$ & $2.772-2$ \\
\hline 19 & $9.546-1$ & $-1.260+1$ & $7.637-\overline{2}$ & $2 \overline{2}$ & 0 & $-2.401+0$ & $1.277+0$ & $2.578-1$ & 25 & 8 & $1.272+0$ & $-9.016+0$ & $1.107-5$ \\
\hline 19 & 7.531-1 & $-1.215+1$ & $8.907-\overline{2}$ & 23 & 23 & $-9.824-3$ & $2.758+0$ & $4.235-1$ & 25 & 7 & $1.925+0$ & $-1.345+1$ & $-2.248-2$ \\
\hline 20 & $.633-1$ & $5.219+0$ & 4.591-1 & 23 & 22 & $-9.558+0$ & $1.929+1$ & $9.715-1$ & 25 & 6 & $-2.282-1$ & $-5.480+0$ & $1.130-1$ \\
\hline 20 & $-1.098+1$ & 2.502 & $1.059+0$ & 23 & 21 & $-1.436+1$ & $4.560+1$ & $1.112+0$ & 25 & 5 & $-6.457-1$ & $-4.666+0$ & $1.450-1$ \\
\hline 20 & $-1.521+1$ & $4.940+1$ & $1.162+0$ & 23 & 20 & $-1.292+1$ & $4.346+1$ & 9.555-1 & 25 & 4 & $-1.152+0$ & $-2.976+0$ & $1.750-1$ \\
\hline 20 & $-1.239+1$ & $4.198+1$ & $9.203-1$ & 23 & 19 & $-9.646+0$ & $3.202+1$ & 7.223-1 & 25 & 3 & $-2.050+0$ & 1.238-1 & $2.314-1$ \\
\hline 20 & $-8.241+0$ & $2.739+1$ & $6.317-1$ & 23 & 18 & $-6.789+0$ & $2.201+1$ & $5.243-1$ & 25 & 2 & $-2.563+0$ & $1.350+0$ & $2.694-1$ \\
\hline 20 & $-5.497+0$ & $1.777+1$ & $4.429-1$ & 23 & 17 & $-4.228+0$ & $1.270+1$ & $3.533-1$ & 25 & 1 & $-3.123+0$ & $3.331+0$ & $3.015-1$ \\
\hline 20 & $-3.261+0$ & $1.049+1$ & 2.843-1 & 23 & 16 & $-2.973+0$ & $8.720+0$ & $2.617-1$ & 25 & 0 & $-4.193+0$ & $7.749+0$ & $3.627-1$ \\
\hline 13 & $-2.594+0$ & $8.942+0$ & 2.300-1 & 23 & 15 & $-2.409+0$ & $6.931+0$ & $2.211-1$ & 26 & 26 & $-1.387+1$ & 4.935 & $1.444+0$ \\
\hline 12 & $-1.520+0$ & $5.498+0$ & $1.526-1$ & 23 & 14 & $-7.813-1$ & $8.482-1$ & $1.175-1$ & 26 & 25 & $-7.943+0$ & 1.354 & 8.644-1 \\
\hline 11 & $-1.832+0$ & $6.523+0$ & 1.742 & 23 & 13 & $-1.076+0$ & $2.474+0$ & $1.298-1$ & 26 & 24 & $-1.320+1$ & 4.158 & $1.031+0$ \\
\hline 10 & $-8.574-1$ & $2.556+0$ & $1.145-1$ & 23 & 12 & $-6.443-1$ & 9.810-1 & $1.012-1$ & 26 & 23 & $-1.143+1$ & 3.753 & 8.595-1 \\
\hline 20 & $-4.627-1$ & 4.657-1 & $9.529-2$ & 23 & 11 & $-1.005+0$ & $2.708+0$ & $1.191-1$ & 26 & 22 & $-8.823+0$ & 2.869 & $6.671-1$ \\
\hline 20 & $5.117-1$ & $-5.050+0$ & $5.345-2$ & 23 & 10 & $-9.677-2$ & $-1.326+0$ & $6.836-2$ & 26 & 21 & $-7.249+0$ & 2.367 & $5.465-1$ \\
\hline 20 & $1.071+0$ & $-8.966+0$ & $3.567-2$ & 23 & 9 & $3.263-1$ & $-3.781+0$ & $5.050-\overline{2}$ & 26 & 20 & $-5.094+0$ & 1.608 & $3.975-1$ \\
\hline 20 & $1.469+0$ & -1.168 & $2.069-2$ & 23 & 8 & $1.434+0$ & $-9.628+0$ & $-3.157-3$ & 26 & 19 & $-4.322+0$ & 1.341 & $3.423-1$ \\
\hline 20 & $1.290+0$ & $-1.197+1$ & $4.029-2$ & 23 & 7 & $1.096+0$ & $-9.616+0$ & $2.996-2$ & 26 & 18 & $-3.213+0$ & 9.331 & $2.686-1$ \\
\hline 20 & $7.764-1$ & $-1.039+1$ & $7.445-2$ & 23 & 6 & $1.243+0$ & $-1.151+1$ & $3.339-2$ & 26 & 17 & $-2.373+0$ & $5.916+0$ & 2.181-1 \\
\hline 20 & $6.572-1$ & $-1.027+1$ & $8.174-2$ & 23 & 5 & 3.603-2 & -7.125 & $1.078-\overline{1}$ & 26 & 16 & $-1.904+0$ & $4.472+0$ & $1.832-1$ \\
\hline 20 & $6.810-1$ & $-1.098+1$ & $8.535-2$ & 23 & 4 & -3.361 & -6.121 & $1.322-1$ & 26 & 15 & $-9.573-1$ & 6.00 & $1.278-1$ \\
\hline 20 & $2.872-1$ & $-9.814+0$ & $1.100-1$ & 23 & 3 & -1.047 & -3.879 & $1.790-1$ & 26 & 14 & $-1.093+0$ & 1.328 & 1.33 \\
\hline 20 & $4.212-2$ & $-8.881+0$ & $1.233-1$ & 23 & 2 & $-2.527+0$ & $1.841+0$ & $2.677-1$ & 26 & 13 & 7.274-1 & $-5.768+0$ & $2.070-2$ \\
\hline 21 & $-7.878+0$ & $3.378+1$ & $9.225-1$ & 23 & $\overline{1}$ & $-2.473+0$ & $1.239+0$ & $2.644-1$ & 26 & 12 & $3.128-1$ & $-3.548+0$ & $3.951-2$ \\
\hline 20 & $-9.218+0$ & $1.746+1$ & $9.570-1$ & 23 & 0 & $-3.711+0$ & $6.484+0$ & $3.337-1$ & 26 & 11 & 4.096-1 & $-3.856+0$ & $3.423-2$ \\
\hline 19 & $-1.562+1$ & 5.082 & $1.189+0$ & 24 & 24 & $-1.686+0$ & 9.535 & $5.241-1$ & 26 & 10 & $-3.330-1$ & -6.37 & $7.490-2$ \\
\hline 18 & $-1.373+1$ & $4.721+1$ & $1.002+0$ & 24 & 23 & $-8.838+0$ & 1.650 & $9.263-1$ & 26 & 9 & $-1.275-1$ & -2.157 & $6.912-2$ \\
\hline 17 & $-8.612+0$ & $2.859+1$ & 6.544-1 & 24 & 22 & $-1.384+1$ & $4.341+1$ & $1.082+0$ & 26 & 8 & $1.077+0$ & $-8.569+0$ & $1.311-2$ \\
\hline 16 & $-5.535+0$ & $1.759+1$ & $4.453-1$ & 24 & 21 & $-1.285+1$ & $4.293+1$ & 9.527-1 & 26 & 7 & $6.734-1$ & $-8.211+0$ & $4.732-2$ \\
\hline 15 & $-4.418+0$ & $1.457+1$ & $3.580-1$ & 24 & 20 & $-9.737+0$ & 3.240 & $7.248-1$ & 26 & 6 & $1.165+0$ & -1.144 & $2.847-2$ \\
\hline 14 & $-2.355+0$ & $7.224+0$ & $2.205-1$ & 24 & 19 & $-5.843+0$ & 1.804 & $4.652-1$ & 26 & 5 & $-7.448-1$ & $-4.167+0$ & $1.452-1$ \\
\hline 13 & $-1.149+0$ & $3.234+0$ & 1.363-1 & 24 & 18 & $-4.838+0$ & $1.481+1$ & 3.909-1 & 26 & 4 & $-2.167+0$ & 8.234-1 & $2.361-1$ \\
\hline 12 & $-9.573-1$ & $2.954+0$ & $1.179-1$ & 24 & 17 & $-3.247+0$ & $9.352+0$ & $2.807-1$ & 26 & 3 & $-2.433+0$ & $1.212+0$ & $2.560-1$ \\
\hline 11 & $-2.255-1$ & $-2.267-1$ & $7.643-\overline{2}$ & 24 & 16 & $-1.965+0$ & $4.887+0$ & $1.937-1$ & 26 & 2 & $-3.349+0$ & $4.369+0$ & $3.129-1$ \\
\hline 10 & $-7.165-1$ & $1.609+0$ & $1.071-1$ & 24 & 15 & $-2.598+0$ & $7.885+0$ & $2.261-1$ & 26 & $\overline{1}$ & $-3.496+0$ & $4.433+0$ & $3.254-1$ \\
\hline 21 & 4.839-1 & $-3.916+0$ & $4.093-2$ & 24 & 14 & $-8.104-1$ & 7.822 & $1.174-1$ & 26 & 0 & $-4.662+0$ & 9.550 & 3.85 \\
\hline 21 & $7.060-1$ & $-5.872+0$ & $3.773-2$ & 24 & 13 & $-3.463-1$ & -7.984-1 & 8.616-2 & 27 & 27 & $-2.936+0$ & $1.454+1$ & $5.965-1$ \\
\hline 21 & $2.335+0$ & -1.437 & $-4.177-2$ & 24 & 12 & $-1.624-1$ & $-1.481+0$ & $7.513-2$ & 27 & 26 & $-8.065+0$ & 1.417 & $8.707-1$ \\
\hline 21 & $1.834+0$ & $-1.348+1$ & $-7.405-4$ & 24 & 11 & $1.995-2$ & -1.738 & $5.818-2$ & 27 & 25 & $-1.300+1$ & 4.066 & $1.022+0$ \\
\hline 21 & $1.218+0$ & $-1.185+1$ & $4.341-2$ & 24 & 10 & $1.787-1$ & -2.640 & $5.058-2$ & 27 & 24 & $-1.162+1$ & 3.878 & 8.654-1 \\
\hline 21 & $-2.594-1$ & $-6.429+0$ & $1.358-1$ & 24 & 9 & 4.413-1 & $-4.404+0$ & $4.205-2$ & 27 & 23 & $-8.677+0$ & 2.842 & $6.524-1$ \\
\hline 21 & 1.894-1 & $-8.276+0$ & $1.040-1$ & 24 & 8 & $1.350+0$ & $-9.247+0$ & $-2.597-3$ & 27 & 22 & $-7.247+0$ & 2.399 & $5.413-1$ \\
\hline 21 & $-5.737-1$ & $-5.933+0$ & $1.576-1$ & 24 & 7 & $1.310+0$ & $-1.066+1$ & $1.596-2$ & 27 & 21 & $-5.611+0$ & 1.816 & $4.271-1$ \\
\hline 21 & $-2.078+0$ & 8.646-2 & $2.450-1$ & 24 & 6 & $1.118+0$ & -1.103 & $3.679-2$ & 27 & 20 & $-5.266+0$ & 1.711 & 3.98 \\
\hline 21 & $-1.380+0$ & $-2.837+0$ & 2.003-1 & 24 & 5 & $9.631-2$ & -7.551 & $1.023-1$ & 27 & 19 & $-4.212+0$ & 1.310 & $3.300-1$ \\
\hline 22 & $-2.469+1$ & $9.351+1$ & $2.096+0$ & 24 & 4 & $-6.671-1$ & $-4.864+0$ & $1.495-1$ & 27 & 18 & $-3.403+0$ & $9.863+0$ & $2.801-1$ \\
\hline 21 & $-9.867+0$ & $2.048+1$ & $9.912-1$ & 24 & 3 & $-1.349+0$ & $-2.408+0$ & $1.896-1$ & 27 & 17 & $-1.619+0$ & $2.685+0$ & $1.705-1$ \\
\hline 20 & $-1.577+1$ & $5.142+1$ & $1.196+0$ & 24 & 2 & $-2.168+0$ & 2.095 & $2.464-1$ & 27 & 16 & $-1.189+0$ & 1.203 & $1.411-1$ \\
\hline 19 & $-1.212+1$ & $4.028+1$ & 9.079 & 24 & 1 & -2.507 & 1.230 & $2.637-1$ & 27 & 15 & $-7.376-1$ & & $1.110-1$ \\
\hline 18 & $-8.594+0$ & $2.822+1$ & $6.542-1$ & 24 & 0 & $-4.264+0$ & $8.588+0$ & 3.643-1 & 27 & 14 & $-4.477-1$ & $-1.544+0$ & $9.486-2$ \\
\hline 17 & $-5.964+0$ & $1.893+1$ & 4.734-1 & 25 & 25 & $-2.408+0$ & $1.197+$ & $5.757-1$ & 27 & 13 & $-3.906-1$ & $-1.587+0$ & $8.919-2$ \\
\hline 16 & $-3.413+0$ & $9.867+0$ & $3.007-1$ & 25 & 24 & $-8.519+0$ & 1.552 & 9.036-1 & 27 & 12 & $1.733-1$ & $-3.522+0$ & $5.170-2$ \\
\hline 15 & $-2.868+0$ & $8.786+0$ & $2.532-$ & 25 & 23 & -1.423 & 4.529 & $1.100+0$ & 27 & 11 & 4.68 & $-4.214+0$ & $2.833-2$ \\
\hline 14 & $-2.101+0$ & $6.302+0$ & $1.992-1$ & 25 & 22 & $-1.201+1$ & $3.988+1$ & $8.962-1$ & 27 & 10 & $-5.457-1$ & -1.615-1 & $8.850-2$ \\
\hline 13 & $-1.432+0$ & $4.312+0$ & $1.503-1$ & 25 & 21 & $-9.447+0$ & $3.126+1$ & $7.05 \overline{3}-1$ & 27 & 9 & $-4.018-1$ & $-1.342+0$ & $8.594-2$ \\
\hline 12 & $-5.934-1$ & $1.144+0$ & $9.752-2$ & 25 & 20 & $-5.849+0$ & $1.800+1$ & 4.643-1 & 27 & 8 & 8.418-1 & $-7.948+0$ & $2.719-2$ \\
\hline 11 & $-5.787-1$ & $1.274+0$ & $9.265-2$ & 25 & 19 & $-5.115+0$ & $1.601+1$ & 4.039-1 & 27 & 7 & $9.086-1$ & $-9.309+0$ & $3.210-2$ \\
\hline 10 & $-4.851-1$ & $5.164-1$ & $9.152-2$ & 25 & 18 & $-3.543+0$ & $1.025+1$ & 2.989-1 & 27 & 6 & $2.521-1$ & $-7.851+0$ & $8.168-2$ \\
\hline 22 & 7.229-1 & $-5.010+0$ & $2.542-2$ & 25 & 17 & $-3.212+0$ & $9.698+0$ & $2.686-1$ & 27 & 5 & $-5.008-1$ & $-5.593+0$ & $1.318-1$ \\
\hline 22 & $1.371+0$ & $-9.158+0$ & $1.270-3$ & 25 & 16 & $-1.805+0$ & $4.208+0$ & $1.800-1$ & 27 & 4 & $-2.256+0$ & $1.288+0$ & $2.359-1$ \\
\hline 22 & $2.660+0$ & $-1.596+1$ & $-6.125-2$ & 25 & 15 & $-9.966-1$ & $1.174+0$ & $1.283-1$ & & 3 & $-2.728+0$ & $2.280+0$ & $2.716-1$ \\
\hline
\end{tabular}

Table 2: Curve fit coefficients of VT transitions rates (continued) 


\begin{tabular}{rlrr}
$\nu_{i}$ & $\nu_{f}$ & $\mathrm{a} 1$ & $\mathrm{a}$ \\
\hline 27 & 2 & $-2.842+0$ & $2.021+0$ \\
27 & 1 & $-3.245+0$ & $3.143+0$ \\
27 & 0 & $-5.298+0$ & $1.147+1$ \\
28 & 28 & $-6.854+0$ & $2.829+1$ \\
28 & 27 & $-6.769+0$ & $9.560+0$ \\
28 & 26 & $-1.156+1$ & $3.520+1$ \\
28 & 25 & $-1.054+1$ & $3.436+1$ \\
28 & 24 & $-8.012+0$ & $2.584+1$ \\
28 & 23 & $-7.328+0$ & $2.456+1$ \\
28 & 22 & $-6.270+0$ & $2.076+1$ \\
28 & 21 & $-4.407+0$ & $1.355+1$ \\
28 & 20 & $-3.926+0$ & $1.212+1$ \\
28 & 19 & $-3.653+0$ & $1.071+1$ \\
28 & 18 & $-2.078+0$ & $4.225+0$ \\
28 & 17 & $-1.776+0$ & $3.020+0$ \\
28 & 16 & $-1.699+0$ & $2.995+0$ \\
28 & 15 & $-3.826-1$ & $-2.205+0$ \\
28 & 14 & $-1.006+0$ & $7.585-1$ \\
28 & 13 & $-5.147-1$ & $-1.081+0$ \\
28 & 12 & $2.755-1$ & $-4.132+0$ \\
28 & 11 & $1.188-1$ & $-3.122+0$ \\
28 & 10 & $-6.571-1$ & $2.862-1$ \\
28 & 9 & $-5.736-1$ & $-8.745-1$ \\
28 & 8 & $6.911-3$ & $-4.604+0$ \\
28 & 7 & $-1.137-1$ & $-5.410+0$ \\
28 & 6 & $4.267-1$ & $-8.670+0$ \\
28 & 5 & $-1.762+0$ & $-4.727-1$ \\
28 & 4 & $-2.246+0$ & $8.279-1$ \\
28 & 3 & $-3.285+0$ & $4.408+0$ \\
28 & 2 & $-2.204+0$ & $-8.758-1$ \\
28 & 1 & $-3.309+0$ & $3.017+0$ \\
28 & 0 & $-6.309+0$ & $1.537+1$ \\
29 & 29 & $-2.011+0$ & $1.093+1$ \\
29 & 28 & $-7.506+0$ & $1.293+1$ \\
29 & 27 & $-1.170+1$ & $3.637+1$ \\
29 & 26 & $-1.069+1$ & $3.547+1$ \\
29 & 25 & $-8.502+0$ & $2.833+1$ \\
29 & 24 & $-6.701+0$ & $2.204+1$ \\
29 & 23 & $-5.675+0$ & $1.854+1$ \\
29 & 22 & $-4.477+0$ & $1.390+1$ \\
29 & 21 & $-3.584+0$ & $1.006+1$ \\
29 & 20 & $-2.956+0$ & $7.775+0$ \\
29 & 19 & $-3.020+0$ & $7.811+0$ \\
29 & 18 & $-1.665+0$ & $2.086+0$ \\
29 & 17 & $-1.345+0$ & $8.559-1$ \\
29 & 16 & $-2.681-1$ & $-2.999+0$ \\
29 & 15 & $-1.094+0$ & $2.698-1$ \\
29 & 14 & $3.402-2$ & $-3.816+0$ \\
29 & 13 & $-3.557-1$ & $-2.087+0$ \\
29 & 12 & $1.871-1$ & $-4.065+0$ \\
29 & 11 & $-7.952-1$ & $6.538-1$ \\
29 & 10 & $-1.548+0$ & $3.513+0$ \\
29 & 9 & $-2.427-1$ & $-2.714+0$ \\
29 & 8 & $-1.239+0$ & $1.735-1$ \\
29 & 7 & $-2.651-1$ & $-4.751+0$ \\
29 & 6 & $-2.053+0$ & $1.368+0$ \\
29 & 5 & $-2.334+0$ & $1.414+0$ \\
29 & 4 & $-3.616+0$ & $6.527+0$ \\
29 & 3 & $-3.576+0$ & $5.201+0$ \\
29 & 2 & $-4.007+0$ & $6.337+0$ \\
29 & 1 & $-4.153+0$ & $6.453+0$ \\
29 & 0 & $-5.800+0$ & $1.279+1$ \\
30 & 30 & $-8.252+0$ & $3.474+1$ \\
30 & 29 & $-5.970+0$ & $7.397+0$ \\
30 & 28 & $-1.066+1$ & $3.256+1$ \\
30 & 27 & $-9.559+0$ & $3.136+1$ \\
30 & 26 & $-7.770+0$ & $2.568+1$ \\
& & & \\
& &
\end{tabular}

2.830-1

3.087-1

4.287-1

8.716-1

7.839-1

9.291-1

7.998-1

6.104-1

5.416-1

4.654-1

3.458-1

3.067-1

2.929-1

1.975-1

1.791-1

1.714-1

9.077-2

1.235-1

9.270-2

4.354-2

4.886-2

9.196-2

9.620-2

7.328-2

9.256-2

6.814-2

2.042-1

2.364-1

3.025-1

2.436-1

3.124-1

4.874-1

5.366-1

8.249-1

9.299-1

8.032-1

6.325-1

5.028-1

4.255-1

3.465-1

2.928-1

2.494-1

2.545-1

1.757-1

1.542-1

8.323-2

1.353-1

6.146-2

8.348-2

4.908-2

9.939-2

1.469-1

7.804-2

1.492-1

9.729-2

2.154-1

2.406-1

3.127-1

3.202-1

3.491-1

3.592-1

4.593-1

9.408-1

7.231-1

8.630-1

7.261-1

5.847-1 \begin{tabular}{rrr}
$\nu_{i}$ & $\nu_{f}$ & $\mathrm{a} 1$ \\
\hline 30 & 25 & $-6.142+0$ \\
30 & 24 & $-4.384+0$
\end{tabular}

$\begin{array}{lll}30 & 24 & -4.384+0 \\ 30 & 22 & -3.016+0 \\ 30 & 21 & -3.703+0\end{array}$

$\begin{array}{lll}30 & 21 & -3.704+0\end{array}$

$\begin{array}{lll}30 & 20 & -2.179+0\end{array}$

$\begin{array}{lll}30 & 19 & -2.127+0\end{array}$

$\begin{array}{lll}30 & 18 & -1.260+0 \\ 30 & 17 & -6.577-1\end{array}$

$30 \quad 16 \quad-9.451-1$

$\begin{array}{lll}30 & 15 & -1.408+0 \\ 30 & 14 & -9.770-1\end{array}$

$30 \quad 14 \quad-9.770-1$

$\begin{array}{lll}30 & 13 & -1.073+0 \\ 30 & 12 & -3.866-1\end{array}$

$\begin{array}{lll}30 & 12 & -3.866-1 \\ 30 & 11 & -6.139-1\end{array}$

$\begin{array}{lll}30 & 10 & -2.735+0\end{array}$

$\begin{array}{lll}30 & 9 & -1.622+0 \\ 30 & 8 & -1.697+0\end{array}$

$\begin{array}{lll}30 & 8 & -1.697+0\end{array}$

$\begin{array}{lll}30 & 7 & -1.680+0 \\ 30 & 6 & -1.961+0\end{array}$

$\begin{array}{lll}30 & 6 & -1.961+0 \\ 30 & 5 & -2.164+0\end{array}$

$\begin{array}{lll}30 & 4 & -4.671+0\end{array}$

$\begin{array}{lll}30 & 3 & -3.480+0\end{array}$

$\begin{array}{lll}30 & 2 & -3.748+0\end{array}$

$\begin{array}{lll}30 & 1 & -3.916+0 \\ 30 & 0 & -6.699+0\end{array}$

$\begin{array}{lll}30 & 0 & -6.699+0\end{array}$

$\begin{array}{lll}31 & 30 & -5.726+0\end{array}$

$\begin{array}{lll}31 & 29 & -1.065+1\end{array}$

$\begin{array}{lll}31 & 28 & -7.935+0\end{array}$

$\begin{array}{lll}31 & 27 & -7.687+0 \\ 31 & 26 & -5.911+0\end{array}$

$\begin{array}{lll}31 & 26 & -5.911+0\end{array}$

$\begin{array}{lll}31 & 25 & -3.788+0\end{array}$

$\begin{array}{lll}31 & 24 & -4.866+0 \\ 31 & 23 & -3.013+0\end{array}$

$\begin{array}{lll}31 & 23 & -3.013+0\end{array}$

$\begin{array}{lll}31 & 22 & -3.805+0 \\ 31 & 21 & -1.689+0\end{array}$

$\begin{array}{lll}31 & 21 & -1.689+0 \\ 31 & 20 & -1.166+0\end{array}$

$\begin{array}{lll}31 & 19 & -7.677-1\end{array}$

$\begin{array}{lll}31 & 18 & -1.439+0 \\ 31 & 17 & -6.905-1\end{array}$

$31 \quad 17 \quad-6.905-1$

$\begin{array}{lll}31 & 16 & -1.649+0 \\ 31 & 15 & -2.002+0\end{array}$

$\begin{array}{lll}31 & 15 & -2.002+0 \\ 31 & 14 & -1.594+0\end{array}$

$\begin{array}{lll}31 & 13 & -1.843+0\end{array}$

$\begin{array}{lll}31 & 12 & -1.421+0\end{array}$

$31 \quad 11-1.303+0$

$\begin{array}{lll}31 & 10 & -4.010+0 \\ 31 & 9 & -1.299+0\end{array}$

$\begin{array}{lll}31 & 9 & -1.299+0 \\ 31 & 8 & -2.019+0\end{array}$

$\begin{array}{lll}31 & 8 & -2.019+0 \\ 31 & 7 & -3.442+0\end{array}$

$\begin{array}{lll}31 & 7 & -3.442+0 \\ 31 & 6 & -2.641+0 \\ 31 & 5 & -5.435+0\end{array}$

$\begin{array}{lll}31 & 4 & -4.172+0 \\ 31 & 3 & -2.995+0\end{array}$

$\begin{array}{lll}31 & 3 & -2.995+0 \\ 31 & 2 & -3.894+0\end{array}$

$\begin{array}{lll}31 & 1 & -4.146+0\end{array}$

$\begin{array}{lll}31 & 0 & -4.678+0\end{array}$

$\begin{array}{lll}32 & 32 & -6.021+0\end{array}$

$\begin{array}{lll}32 & 31 & -5.535+0\end{array}$

$\begin{array}{lll}32 & 30 & -1.025+1\end{array}$

$\begin{array}{lll}32 & 29 & -8.645+0\end{array}$

$\begin{array}{lll}32 & 28 & -6.132+0\end{array}$

$\begin{array}{lll}32 & 27 & -6.503+0\end{array}$

$\begin{array}{lll}32 & 26 & -4.108+0\end{array}$

$\begin{array}{lll}32 & 25 & -3.226+0 \\ 32 & 24 & -3.435+0\end{array}$ a2

$2.013+1 \quad 4.629-1$

$1.357+1 \quad 3.427-1$

$1.222+1 \quad 3.144-1$

$8.564+0$

2.732-1

$1.044+1$

2.952-1

$4.207+0$

2.025-1

$\begin{array}{rr}8.309-2 & 1.506-1 \\ -2.117+0 & 1.101-1\end{array}$

-4.696-1 1.234-

$1.744+0 \quad 1.473-1$

3.179-1 1.178-1

8.741-1

$-1.628+0$

$-7.260-1$

$1.224-1$

$7.894+0$

7.773-2

$2.769+0 \quad 1.584-1$

$2.071+0 \quad 1.713-1$

9.295-1 1.776-

9.184-1 2.049-1

8.591-1 2.226-1

$1.066+1 \quad 3.726-1$

$4.660+0 \quad 3.104-1$

$4.771+0 \quad 3.343-1$

$4.961+0 \quad 3.470-1$

$1.638+1 \quad 5.091-1$

$9.880+0 \quad 5.223-1$

$6.726+0 \quad 7.050-1$

$3.338+1 \quad 8.517-1$

$2.503+1 \quad 6.255-1$

$2.595+1 \quad 5.703-1$

$1.943+1 \quad 4.451-1$

$1.114+1 \quad 3.052-1$

$1.599+1 \quad 3.597-1$

$8.206+0 \quad 2.463-1$

$1.088+1 \quad 2.999-1$

$1.714+0 \quad 1.767-1$

-2.644-1 1.413-1

$-2.010+0$

1.179-1

$\begin{array}{rr}7.504-1 & 1.575-1 \\ -2.139+0 & 1.104-1\end{array}$

$1.912+0 \quad 1.675-1$

$3.823+0 \quad 1.832-1$

$2.486+0 \quad 1.549-1$

$3.759+0$

$2.127+0$

$1.471+0$

$1.260+1$

$1.183+0$

$3.371+0$

$7.725+0$

$3.338+0$

$1.398+1$

$8.140+0$

$2.182+0$

$5.104+0$

$6.122+0$

$7.188+0$

$2.304+1$

$6.908+0$

$3.201+1$

$2.872+1$

$2.025+1$

$2.230+1$

$1.270+1$

$9.063+0$
$9.835+0$
1.672-1

1.416- 1

1.379-1

2.989-1

1.369-1

1.860-1

2.849-1

2.459-1

4.182-1

3.447-1

2.842-1

3.418-1

3.537-1

3.943-1

8.502-1

6.820-

8.255-1

6.586-1

4.685-1

4.732-1

3.185-1

$2.618-1$

\begin{tabular}{rrrrr}
$\nu_{i}$ & $\nu_{f}$ & $\mathrm{a} 1$ & $\mathrm{a} 2$ & $\mathrm{a} 3$ \\
\hline 32 & 23 & $-2.603+0$ & $6.280+0$ & $2.178-1$
\end{tabular}

$\begin{array}{lllll}32 & 22 & -1.747+0 & 2.329+0 & 1.724-1\end{array}$

$\begin{array}{lllll}32 & 21 & -1.565+0 & 1.083+0 & 1.656-1\end{array}$

$\begin{array}{lllll}32 & 20 & -7.203-1 & -2.380+0 & 1.129-1\end{array}$

$\begin{array}{lllll}32 & 19 & -2.080+0 & 3.103+0 & 1.949-1\end{array}$

$\begin{array}{lllll}32 & 18 & -1.696+0 & 1.818+0 & 1.674-1\end{array}$

$\begin{array}{lllll}32 & 17 & -1.842+0 & 2.280+0 & 1.778-1\end{array}$

$\begin{array}{lllll}32 & 16 & -1.697+0 & 2.091+0 & 1.641-1\end{array}$

$\begin{array}{lllll}32 & 15 & -1.412+0 & 1.305+0 & 1.441-1\end{array}$

$\begin{array}{lllll}32 & 14 & -1.630+0 & 2.511+0 & 1.544-1\end{array}$

$\begin{array}{lllll}32 & 13 & -2.151+0 & 4.846+0 & 1.842-1\end{array}$

$\begin{array}{lllll}32 & 12 & -2.383+0 & 6.153+0 & 1.916-1\end{array}$

$\begin{array}{lllll}32 & 11 & -1.573+0 & 2.513+0 & 1.483-1\end{array}$

$\begin{array}{lllll}32 & 10 & -1.781+0 & 3.133+0 & 1.630-1\end{array}$

$\begin{array}{lllll}32 & 9 & -1.578+0 & 2.010+0 & 1.520-1\end{array}$

$\begin{array}{lllll}32 & 8 & -2.726+0 & 5.401+0 & 2.341-1\end{array}$

$\begin{array}{lll}32 & 7 & -3.633+0\end{array}$

$\begin{array}{lll}32 & 6 & -4.218+0\end{array}$

$\begin{array}{lll}32 & 5 & -5.117+0\end{array}$

$\begin{array}{lll}32 & 4 & -4.937+0\end{array}$

$\begin{array}{lll}32 & 3 & -6.064+0\end{array}$

$\begin{array}{lll}32 & 2 & -4.180+0\end{array}$

$\begin{array}{lll}32 & 1 & -5.268+0\end{array}$

$\begin{array}{lll}32 & 0 & -5.784+0\end{array}$

$\begin{array}{lll}33 & 33 & -6.409+0\end{array}$

$\begin{array}{lll}33 & 32 & -5.376+0\end{array}$

$\begin{array}{lll}33 & 31 & -1.068+1\end{array}$

$\begin{array}{lll}33 & 30 & -7.146+0\end{array}$

$\begin{array}{lll}33 & 29 & -5.028+0\end{array}$

$\begin{array}{lll}33 & 28 & -5.455+0\end{array}$

$\begin{array}{lll}33 & 27 & -3.524+0\end{array}$

$\begin{array}{lll}33 & 26 & -2.848+0\end{array}$

$\begin{array}{lll}33 & 25 & -1.079+0\end{array}$

$\begin{array}{lll}33 & 24 & -1.047+0\end{array}$

$\begin{array}{lll}33 & 23 & -1.599+0\end{array}$

$\begin{array}{lll}33 & 22 & -2.431+0\end{array}$

$\begin{array}{lll}33 & 21 & -2.525+0\end{array}$

$\begin{array}{lll}33 & 20 & -1.469+0\end{array}$

$\begin{array}{lll}33 & 19 & -1.722+0\end{array}$

$\begin{array}{lll}33 & 18 & -2.105+0\end{array}$

$\begin{array}{lll}33 & 17 & -2.495+0\end{array}$

$\begin{array}{lll}33 & 16 & -1.201+0\end{array}$

$\begin{array}{lll}33 & 15 & -3.028+0\end{array}$

$\begin{array}{lll}33 & 14 & -2.861+0\end{array}$

$\begin{array}{lll}33 & 13 & -4.563-1\end{array}$

$\begin{array}{lll}33 & 12 & -7.781-1\end{array}$

$\begin{array}{lll}33 & 11 & -3.043+0\end{array}$

$\begin{array}{lll}33 & 10 & -2.348+0\end{array}$

$\begin{array}{lll}33 & 9 & -4.662+0\end{array}$

$\begin{array}{lll}33 & 8 & -4.473+0\end{array}$

$\begin{array}{lll}33 & 7 & -3.528+0\end{array}$

$\begin{array}{lll}33 & 6 & -6.848+0\end{array}$

$\begin{array}{lll}33 & 5 & -4.759+0\end{array}$

$\begin{array}{lll}33 & 4 & -5.582+0\end{array}$

$\begin{array}{lll}33 & 2 & -4.596+0\end{array}$

$\begin{array}{lll}33 & 1 & -4.734+0\end{array}$

$\begin{array}{lll}33 & 0 & -5.382+0\end{array}$

$\begin{array}{lll}34 & 34 & -4.532+0\end{array}$

$\begin{array}{lll}34 & 33 & -5.617+0\end{array}$

$\begin{array}{lll}34 & 32 & -1.031+1\end{array}$

$\begin{array}{lll}34 & 31 & -8.016+0\end{array}$

$\begin{array}{lll}34 & 30 & -7.373+0\end{array}$

$\begin{array}{lll}34 & 29 & -4.392+0\end{array}$

$\begin{array}{lll}34 & 28 & -2.623+0\end{array}$

$\begin{array}{lll}34 & 27 & -2.315+0\end{array}$

$\begin{array}{lll}34 & 26 & -2.310+0\end{array}$

$8.284+0$

2.942-1

$9.856+0$ 3.325-1

$1.301+1$ 3.885-1

$1.120+1 \quad 3.867-1$ 


\begin{tabular}{|c|c|c|c|c|c|c|c|c|c|c|c|c|c|}
\hline$\nu_{f}$ & a1 & $\mathrm{a} 2$ & $\mathrm{a} 3$ & $\nu_{i}$ & $\nu_{f}$ & a1 & $\mathrm{a} 2$ & a3 & $\nu_{i}$ & $\nu_{f}$ & $\mathrm{a} 1$ & $\mathrm{a} 2$ & $\mathrm{a} 3$ \\
\hline 24 & -1.363 & 5.04 & $1.453-1$ & 36 & 30 & $-2.424+0$ & 6.917 & 1.991-1 & 37 & 1 & -6.830 & 1.593 & 4.922 \\
\hline 23 & $-1.341+0$ & -1.3 & $1.476-1$ & 36 & 29 & $.124-1$ & -5.147 & $1.112-2$ & 37 & 0 & -5.082 & 8.276 & $3.894-1$ \\
\hline 34 & $-1.508+0$ & 7.172 & $1.542-1$ & 36 & 28 & $6.667-2$ & $-4.427+0$ & $4.838-2$ & 38 & 38 & $9.876+0$ & $-3.777-$ & $-1.011-2$ \\
\hline 21 & $-2.735+0$ & $5.380+0$ & $2.308-1$ & 36 & 27 & $-7.384-1$ & $-2.009+0$ & $1.036-\overline{1}$ & 38 & 37 & $-9.767+0$ & 3.122 & 8.515-1 \\
\hline 20 & $-1.660+0$ & 8.587-1 & $1.663-1$ & 36 & 26 & $-4.752-1$ & $-3.902+0$ & $9.562-2$ & 38 & 36 & $-1.184+1$ & 4.489 & $8.375-1$ \\
\hline 19 & $-1.916+0$ & $2.064+0$ & $1.790-1$ & 36 & 25 & & -8.114 & $1.470-2$ & 38 & 35 & $-9.338+0$ & 3.671 & $6.305-1$ \\
\hline 34 & $-1.770+0$ & $1.267+0$ & $1.728-1$ & 36 & 24 & $-5.771-2$ & $-5.751+0$ & $6.163-2$ & 38 & 34 & $-5.196+0$ & 2.012 & $3.570-1$ \\
\hline 34 & $-1.638+0$ & 8.953-1 & $1.645-1$ & 36 & 23 & $2.079+0$ & -1.513 & $-6.193-2$ & 38 & 33 & $-1.455+0$ & 4.775 & $1.208-1$ \\
\hline 16 & $-3.728+0$ & $9.723+0$ & $2.875-1$ & 36 & 22 & $-3.402+0$ & $7.181+0$ & $2.706-1$ & 38 & 32 & $-1.479-2$ & -1.941 & $3.525-2$ \\
\hline 15 & $-2.328+0$ & $4.454+0$ & $1.968-1$ & 36 & 21 & $-3.324+0$ & 7.021 & $2.606-1$ & 38 & 31 & $2.141+0$ & -1.160 & $-9.455-2$ \\
\hline 14 & $-2.891+0$ & $7.127+0$ & $2.264-1$ & 36 & 20 & $-2.449+0$ & $3.247+0$ & 2.091-1 & 38 & 30 & 7.570 & -7.002 & $-2.785-3$ \\
\hline 13 & $-2.273+0$ & $4.673+0$ & $1.895-1$ & 36 & 19 & $-2.769+0$ & $4.889+0$ & $2.229-1$ & 38 & 29 & $3.377+0$ & $-1.813+1$ & $-1.656-1$ \\
\hline 12 & $-2.391+0$ & $5.321+0$ & $1.938-1$ & 36 & 18 & $-3.158+0$ & $6.393+0$ & $2.482-1$ & 38 & 28 & $4.520+0$ & -2.397 & $-2.218-1$ \\
\hline 11 & $-4.920+0$ & 1.551 & $3.469-1$ & 36 & 17 & $-3.040+0$ & $6.005+0$ & $2.421-1$ & 38 & 27 & $3.205+0$ & -1.880 & $-1.477-1$ \\
\hline 10 & $-2.536+0$ & $5.524+0$ & $2.065-1$ & 36 & 16 & $-2.015+0$ & 1.953 & $1.796-1$ & 38 & 26 & 1.634 & -1.318 & $-4.672-2$ \\
\hline 34 & $-2.807+0$ & $5.824+0$ & $2.311-1$ & 36 & 15 & $-3.091+0$ & 6.827 & $2.397-1$ & 38 & 25 & $1.049+0$ & -1.099 & $-1.127-2$ \\
\hline 34 & $-4.390+0$ & $1.145+1$ & $3.328-1$ & 36 & 14 & $-2.751+0$ & 5.723 & 2.161-1 & 38 & 24 & $1.008+0$ & -1.137 & $-4.203-3$ \\
\hline 34 & $-5.251+0$ & 1.415 & $3.902-1$ & 36 & 13 & $-4.800+0$ & 1.417 & $3.392-1$ & 38 & 23 & -1.279 & -2.244 & $1.334-1$ \\
\hline 34 & -3.495 & 6.187 & $2.883-1$ & 36 & 12 & $-3.964+0$ & 1.080 & $2.886-1$ & 38 & 22 & 5.30 & -9.825 & $2.567-2$ \\
\hline 34 & $-6.947+0$ & $1.922+1$ & $5.046-1$ & 36 & 11 & $-2.587+0$ & $4.738+0$ & $2.095-1$ & 38 & 21 & 1.658 & -9.018 & $5.519-2$ \\
\hline 34 & $-7.177+0$ & $1.958+1$ & $5.195-1$ & 36 & 10 & $-2.436+0$ & $4.060+0$ & $2.002-1$ & 38 & 20 & $-9.151-1$ & -3.799 & $1.090-1$ \\
\hline 34 & $-5.691+0$ & $1.248+1$ & $4.376-1$ & 36 & 9 & $-5.756+0$ & $1.754+1$ & $3.970-1$ & 38 & 19 & $-1.741+0$ & -1.122 & $1.669-1$ \\
\hline 34 & $-5.309+0$ & 1.066 & 4.143-1 & 36 & 8 & -4.537 & 1.130 & $3.370-1$ & 38 & 18 & $-1.131+0$ & -2.849 & $1.205-1$ \\
\hline 34 & $-3.328+0$ & $2.308+0$ & $2.953-1$ & 36 & 7 & $-5.799+0$ & 1.613 & 4.103-1 & 38 & 17 & $-1.958+0$ & 7.16 & $1.703-1$ \\
\hline 34 & $-5.875+0$ & $1.200+1$ & $4.525-1$ & 36 & 6 & $-5.701+0$ & 1.471 & $4.128-1$ & 38 & 16 & $-2.156+0$ & 1.986 & $1.781-1$ \\
\hline 35 & 1.409 & -6.296 & $-2.376-1$ & 36 & 5 & $-5.578+0$ & 1.365 & $4.058-1$ & 38 & 15 & $-8.241-1$ & -3.804 & $1.043-1$ \\
\hline 34 & $-5.402+0$ & $9.525+0$ & $6.352-1$ & 36 & 4 & $-4.931+0$ & 9.549 & $3.802-1$ & 38 & 14 & $-3.473-1$ & -5.139 & $6.851-2$ \\
\hline 33 & $-1.037+1$ & 3.554 & $7.940-1$ & 36 & 3 & $-4.623+0$ & 7.715 & $3.653-1$ & 38 & 13 & -3.066 & 6.274 & $2.283-1$ \\
\hline 35 & $-7.896+0$ & $2.801+1$ & $5.822-1$ & 36 & 2 & $-5.644+0$ & 1.122 & $4.303-1$ & 38 & 12 & -3.249 & 6.430 & $2.469-1$ \\
\hline 35 & $-6.193+0$ & $2.188+1$ & $4.520-1$ & 36 & 1 & $-6.875+0$ & 1.654 & $4.986-1$ & 38 & 11 & $-2.709+0$ & 4.772 & $2.060-1$ \\
\hline 30 & $-3.316+0$ & $1.046+1$ & $2.648-1$ & 36 & 0 & $-5.968+0$ & 1.223 & $4.473-1$ & 38 & 10 & $-3.432+0$ & 7.195 & 2.533-1 \\
\hline 29 & -2.713 & 8.285 & $2.135-1$ & 37 & 37 & 1.214 & -5.20 & $-1.688-1$ & 38 & 9 & -5.763 & 1.593 & $4.010-1$ \\
\hline 28 & $-2.091+0$ & $4.650+0$ & $1.848-1$ & 37 & 36 & $-7.736+0$ & 2.155 & $7.469-1$ & 38 & 8 & $-4.620+0$ & 1.044 & $3.401-1$ \\
\hline 27 & $-7.684-1$ & $-1.066+0$ & $1.025-1$ & 37 & 35 & $-1.240+1$ & $4.614+1$ & $8.848-1$ & 38 & 7 & $-6.586+0$ & 1.817 & $4.532-1$ \\
\hline 26 & $-4.030-1$ & $-3.122+0$ & $8.357-2$ & 37 & 34 & $-7.454+0$ & $2.799+1$ & $5.308-1$ & 38 & 6 & $-7.409+0$ & 2.051 & $5.125-1$ \\
\hline 25 & 4.573 & $-7.170+0$ & $3.325-2$ & 37 & 33 & -5.262 & 1.981 & $3.730-1$ & 38 & 5 & -5.230 & 1.040 & $3.895-1$ \\
\hline 24 & -4.91 & -3.521 & $8.956-2$ & 37 & 32 & $-3.917+0$ & 1.421 & $2.803-1$ & 38 & 4 & -6.901 & 1.652 & $4.943-1$ \\
\hline 23 & -7.939 & $-2.578+0$ & $1.097-1$ & 37 & 31 & $-1.783+0$ & $4.774+0$ & $1.496-1$ & 38 & 3 & -6.512 & 1.415 & $4.765-1$ \\
\hline 22 & $-2.593+0$ & $4.344+0$ & $2.216-1$ & 37 & 30 & 2.296-1 & $-4.131+0$ & $3.017-2$ & 38 & 2 & $-4.452+0$ & 5.279 & $3.538-1$ \\
\hline 21 & $-2.471+0$ & $3.806+0$ & $2.131-1$ & 37 & 29 & $1.074+0$ & $-8.051+0$ & $-2.367-2$ & 38 & 1 & $-6.047+0$ & 1.299 & $4.342-1$ \\
\hline 20 & -7.77 & -3.277 & $1.129-1$ & 37 & 28 & 1.281 & -9.963 & $-2.731-2$ & 38 & 0 & $-2.160+0$ & $-3.408+0$ & $2.020-1$ \\
\hline 19 & $-1.485+0$ & -2.63 & $1.534-1$ & 37 & 27 & $1.444+0$ & -1.119 & $-3.505-2$ & 39 & 39 & 2.512 & -9.352 & $3.057-1$ \\
\hline 18 & $-1.601+0$ & 3.671 & $1.567-1$ & 37 & 26 & $2.390+0$ & $-1.612+1$ & $-8.111-2$ & 39 & 38 & -1.029 & 3.435 & $8.758-1$ \\
\hline 17 & $-2.112+0$ & 2.770 & $1.845-1$ & 37 & 25 & $-4.614-1$ & $-4.248+0$ & $8.286-2$ & 39 & 37 & $-1.430+1$ & 5.646 & $9.654-1$ \\
\hline 16 & -2.182 & 3.264 & $1.877-1$ & 37 & 24 & -1.022 & -2.225 & $1.164-1$ & 39 & 36 & $-9.118+0$ & 3.673 & $6.046-1$ \\
\hline 15 & $-4.594+0$ & $1.354+1$ & $3.280-1$ & 37 & 23 & $-1.773+0$ & 2.23 & $1.674-1$ & 39 & 35 & -3.952 & 1.590 & $2.729-1$ \\
\hline 14 & $-3.477+0$ & $9.374+0$ & $2.561-1$ & 37 & 22 & $-5.971-1$ & $-4.900+0$ & $9.977-2$ & 39 & 34 & $-2.413+0$ & 9.103 & $1.709-1$ \\
\hline 13 & $-5.766+0$ & $1.911+1$ & $3.904-1$ & 37 & 21 & $-6.526-2$ & $-6.793+0$ & $6.080-\overline{2}$ & 39 & 33 & $-2.362-1$ & $-7.707-1$ & $4.202-2$ \\
\hline 12 & $-3.133+0$ & $7.794+0$ & $2.393-1$ & 37 & 20 & $-2.727+\overline{0}$ & $4.087+0$ & $2.207-1$ & 3 & 32 & $2.317+0$ & -1.215 & $-1.088-\overline{1}$ \\
\hline 11 & $-2.993+0$ & $6.948+0$ & $2.339-1$ & 37 & 19 & $-2.177+0$ & 1.638 & $1.908-1$ & 39 & 31 & 4.802 & -2.309 & $-2.590-1$ \\
\hline 10 & $-2.343+0$ & $4.501+0$ & $1.908-1$ & 37 & 18 & $-2.665+0$ & 3.817 & $2.174-1$ & 39 & 30 & 4.205 & -2.212 & $-2.103-1$ \\
\hline 35 & $-6.051+0$ & $1.898+1$ & 4.191-1 & 37 & 17 & $-7.593-1$ & $-3.834+0$ & $1.034-1$ & 39 & 29 & $4.792+0$ & -2.490 & $-2.490-1$ \\
\hline 35 & $-4.078+0$ & $9.639+0$ & $3.135-1$ & 37 & 16 & $-6.537-1$ & $-4.213+0$ & $9.806-2$ & 39 & 28 & $4.161+0$ & -2.313 & $-2.033-1$ \\
\hline 35 & $-5.043+0$ & $1.303-$ & $3.722-1$ & 37 & 15 & $-2.468+0$ & 3.695 & $2.025-1$ & 3 & 27 & $4.063+0$ & -2.310 & $-1.999-1$ \\
\hline 35 & $-5.700+0$ & $1.509+1$ & $4.156-1$ & 37 & 14 & $-2.942+0$ & 6.038 & 2.255 & 39 & 26 & $3.512+0$ & -2.146 & $-1.604-1$ \\
\hline 35 & $-5.753+0$ & $1.427+1$ & $4.252-1$ & 37 & 13 & $-2.697+0$ & $5.183+0$ & $2.104-1$ & 39 & 25 & $3.771+0$ & -2.305 & $-1.728-1$ \\
\hline 35 & $-5.183+0$ & $1.121+1$ & $3.942-1$ & 37 & 12 & $-3.754+0$ & $9.673+0$ & $2.709-1$ & 39 & 24 & $2.355+0$ & $-1.766+1$ & $-8.541-2$ \\
\hline 35 & $-5.636+0$ & 1.192 & $4.315-1$ & 37 & 11 & -3.712 & 9.417 & 2.694-1 & 39 & 23 & $3.689+0$ & $-2.347+1$ & $-1.657-\overline{1}$ \\
\hline 35 & $-7.374+0$ & $1.905+1$ & $5.311-1$ & 37 & 10 & $-3.517+0$ & 7.648 & $2.683-1$ & 39 & 22 & $3.394-1$ & $-9.871+0$ & $3.931-2$ \\
\hline 35 & $-5.257+0$ & $1.008+1$ & $4.046-1$ & 37 & 9 & $-3.818+0$ & $8.617+0$ & $2.863-1$ & 39 & 21 & $1.706+0$ & $-1.607+1$ & $-3.679-2$ \\
\hline 35 & $-5.623+0$ & $1.072+1$ & $4.346-1$ & 37 & 8 & $-4.527+0$ & $1.091+1$ & 3.313-1 & 39 & 20 & $-8.844-1$ & $-5.169+0$ & 1.149-1 \\
\hline 36 & $1.363+1$ & -5.891 & $-2.581-1$ & 37 & 7 & $-7.753+0$ & $2.349+1$ & $5.289-1$ & 39 & 19 & $-3.914-1$ & $-7.406+0$ & 8.849-2 \\
\hline 35 & $-6.789+0$ & $1.645+1$ & $7.052-1$ & 37 & 6 & $-6.814+0$ & $1.816+1$ & $4.855-1$ & 39 & 18 & $2.368-1$ & $-9.701+0$ & $4.763-2$ \\
\hline 34 & $-1.067+1$ & $3.783+1$ & $7.964-1$ & 37 & 5 & $-4.781+0$ & $9.751+0$ & $3.580-1$ & 39 & 17 & $-6.565-1$ & $-5.729+0$ & $9.817-2$ \\
\hline 33 & $-8.936+0$ & $3.298+1$ & $6.349-1$ & 37 & 4 & $-8.642+0$ & $2.419+1$ & 6.033-1 & 39 & 16 & $-8.576-2$ & $-7.633+0$ & $6.065-2$ \\
\hline 32 & $-5.199+0$ & $1.863+1$ & $3.816-1$ & 37 & 3 & $-5.675+0$ & $1.206+1$ & 4.192-1 & 39 & 15 & 8.095-1 & $-1.070+1$ & $1.155-\overline{3}$ \\
\hline 31 & $-4.051+0$ & 1.442 & 2.937-1 & 37 & 2 & $-4.735+0$ & $7.241+0$ & 3.699-1 & 39 & 14 & $-1.134+0$ & $-2.552+0$ & $1.162-1$ \\
\hline
\end{tabular}

Table 2: Curve fit coefficients of VT transitions rates (continued) 


\begin{tabular}{|c|c|c|c|c|c|c|c|c|c|c|c|c|c|c|}
\hline$\nu_{i}$ & $\nu_{f}$ & a1 & $\mathrm{a} 2$ & a.3 & $\nu_{i}$ & $\nu_{f}$ & a1 & $\mathrm{a} 2$ & a.3 & $\nu_{i}$ & $\nu_{f}$ & a1 & $\mathrm{a} 2$ & a3 \\
\hline 39 & 13 & -1.233 & $-2.018+0$ & $1.212-1$ & 41 & 29 & 6.522 & -3.305 & $-3.718-1$ & 42 & 5 & $-5.423+0$ & 9.269 & $3.584-1$ \\
\hline 39 & 12 & -4.79 & $-5.207+0$ & $7.639-2$ & 41 & 28 & 7.055 & $-3.606+1$ & $-3.956-1$ & 42 & 4 & $-4.773+0$ & 5.529 & $3.304-1$ \\
\hline 39 & 11 & $-1.653+0$ & $-4.503-1$ & $1.467-1$ & 41 & 27 & $4.463+0$ & $-2.612+1$ & $-2.352-1$ & 42 & 3 & $-4.249+0$ & $3.630+0$ & 2.898-1 \\
\hline 39 & 10 & $1.170-1$ & $-8.666+0$ & $5.092-2$ & 41 & 26 & $5.401+0$ & $-3.054+1$ & $-2.872-1$ & 42 & 2 & $-5.210+0$ & $7.486+0$ & $3.473-1$ \\
\hline 39 & 9 & $-2.829+0$ & $3.358+0$ & $2.235-1$ & 41 & 25 & $5.700+0$ & -3.223 & $-3.024-1$ & 42 & 1 & $-5.620+0$ & 9.386 & 3.689-1 \\
\hline 39 & 8 & $-7.191+0$ & $2.026+1$ & $4.946-1$ & 41 & 24 & $3.913+0$ & -2.507 & $-1.952-1$ & 42 & 0 & $-6.618+0$ & 1.346 & $4.280-1$ \\
\hline 39 & 7 & $-3.490+0$ & $4.554+0$ & $2.719-1$ & 41 & 23 & $4.950+0$ & $-2.955+1$ & $-2.563-1$ & 43 & 43 & $-3.813+0$ & 2.035 & $6.874-1$ \\
\hline 39 & 6 & $-4.957+0$ & $1.013+1$ & 3.593-1 & 41 & 22 & $2.736+0$ & $-2.085+1$ & $-1.186-1$ & 43 & 42 & $-9.478+0$ & 3.467 & 7.997-1 \\
\hline 39 & 5 & $-7.675+0$ & 2.028 & $5.307-1$ & 41 & 21 & 3.714 & -2.522 & $-1.746-1$ & 43 & 41 & -1.222 & 4.957 & 8.033-1 \\
\hline 39 & 4 & $-7.013+0$ & $1.643+1$ & $5.012-1$ & 41 & 20 & $5.907+0$ & -3.388 & $-3.110-1$ & 43 & 40 & -1.034 & 4.210 & $6.570-1$ \\
\hline 39 & 3 & $-5.404+0$ & $9.188+0$ & 4.083-1 & 41 & 19 & $1.428+0$ & -1.595 & $-3.571-2$ & 43 & 39 & $-9.103+0$ & 3.665 & 5.518-1 \\
\hline 39 & 2 & $-4.581+0$ & $5.640+0$ & $3.561-1$ & 41 & 18 & $2.848+0$ & -2.155 & $-1.230-1$ & 43 & 38 & $-7.092+0$ & 2.717 & 4.323-1 \\
\hline 39 & 1 & $-7.923+0$ & $2.024+1$ & 5.420 & 41 & 17 & $2.860+0$ & -2.145 & $-1.252-1$ & 43 & 37 & $-6.104+0$ & 2.231 & $3.601-1$ \\
\hline 39 & 0 & $-3.469+0$ & 1.625 & $2.767-$ & 41 & 16 & 2.55 & -9.979 & $2.607-2$ & 43 & 36 & $-1.855+0$ & 3.897 & $1.052-1$ \\
\hline 40 & 40 & $-9.760-4$ & $2.431+0$ & 4.813-1 & 41 & 15 & $3.321+0$ & $-2.195+1$ & $-1.637-1$ & 43 & 35 & $-8.243-2$ & -3.662 & $-9.612-3$ \\
\hline 40 & 39 & $-1.150+1$ & $4.061+1$ & $9.302-1$ & 41 & 14 & $1.576+0$ & $-1.482+1$ & $-5.558-2$ & 43 & 34 & $2.220+0$ & -1.302 & $-1.648-1$ \\
\hline 40 & 38 & $-1.460+1$ & $5.856+1$ & $9.734-1$ & 41 & 13 & $1.881+0$ & -1.594 & $-7.526-2$ & 43 & 33 & $3.198+0$ & -1.848 & $-2.059-1$ \\
\hline 40 & 37 & $-1.060+1$ & 4.359 & 6.804-1 & 41 & 12 & 1.649 & -1.529 & $-6.010-2$ & 43 & 32 & $3.753+0$ & -2.143 & $-2.388-1$ \\
\hline 40 & 36 & $-7.534+0$ & $3.123+$ & $4.736-1$ & 41 & 11 & $-1.044+0$ & $-4.267+0$ & $9.804-2$ & 43 & 31 & $6.119+0$ & -3.106 & $-3.929-1$ \\
\hline 40 & 35 & $-2.914+0$ & $1.120+1$ & $1.958-1$ & 41 & 10 & $-4.479-1$ & $-6.696+0$ & $5.896-2$ & 43 & 30 & $7.803+0$ & -3.967 & $-4.732-1$ \\
\hline 40 & 34 & $-1.615+0$ & $5.780+0$ & $1.043-1$ & 41 & 9 & $-2.306+0$ & 2.045-1 & $1.746-1$ & 43 & 29 & $7.990+0$ & -4.089 & $-4.845-1$ \\
\hline 40 & 33 & $2.249+0$ & -1.187 & $-1.123-1$ & 41 & 8 & $-4.275+0$ & $8.130+0$ & 2.893-1 & 43 & 28 & $7.904+0$ & -4.136 & $-4.743-1$ \\
\hline 40 & 32 & $5.376+0$ & -2.539 & $-3.019-1$ & 41 & 7 & -4.248 & 7.335 & $2.909-1$ & 43 & 27 & $7.940+0$ & -4.145 & $-4.817-1$ \\
\hline 40 & 31 & $4.519+0$ & -2.262 & $-2.512-1$ & 41 & 6 & $-5.605+0$ & $1.160+1$ & $3.849-1$ & 43 & 26 & $7.407+0$ & -4.024 & $-4.403-1$ \\
\hline 40 & 30 & $5.353+0$ & $-2.754+1$ & $-2.844-1$ & 41 & 5 & $-4.174+0$ & $4.808+0$ & $3.027-1$ & 43 & 25 & $7.019+0$ & -3.911 & $-4.135-1$ \\
\hline 40 & 29 & $5.287+0$ & $-2.739+1$ & $-2.884-1$ & 41 & 4 & $-4.762+0$ & $6.263+0$ & $3.487-1$ & 43 & 24 & $8.207+0$ & -4.412 & $-4.861-1$ \\
\hline 40 & 28 & $5.461+0$ & -2.890 & $-2.907-1$ & 41 & 3 & $-7.403+0$ & 1.640 & $5.108-1$ & 43 & 23 & $6.202+0$ & -3.611 & $-3.653-1$ \\
\hline 40 & 27 & $6.030+0$ & $-3.195+$ & $-3.228-1$ & 41 & 2 & $-6.148+0$ & 1.131 & 4.338-1 & 43 & 22 & $4.526+0$ & -2.986 & $-2.562-1$ \\
\hline 40 & 26 & $3.251+0$ & $-2.092+1$ & $-1.532-1$ & 41 & $\overline{1}$ & $-5.223+0$ & $7.775+0$ & $3.732-1$ & 43 & 21 & $4.039+0$ & -2.708 & $-2.419-1$ \\
\hline 40 & 25 & $4.445+0$ & $-2.616+1$ & $-2.259-1$ & 41 & 0 & $-6.466+0$ & 1.286 & 4. $454-1$ & 43 & 20 & $3.922+0$ & -2.707 & $-2.302-1$ \\
\hline 40 & 24 & $2.659+0$ & -1.951 & $-1.103-1$ & 42 & 42 & 2.14 & 5.072 & 4.611-1 & 43 & 19 & $9.806-1$ & -1.479 & $-5.687-2$ \\
\hline 40 & 23 & $1.476+0$ & -1.453 & $-4.292-2$ & 42 & 41 & -1.246 & 4.640 & $9.705-1$ & 43 & 18 & $3.922+0$ & -2.70 & $-2.294-1$ \\
\hline 40 & 22 & $2.973+0$ & $-2.150+1$ & $-1.248-1$ & 42 & 40 & -1.449 & 5.953 & $9.457-1$ & 43 & 17 & $6.288-1$ & -1.335 & $-3.478-2$ \\
\hline 40 & 21 & $1.203+0$ & $-1.436+1$ & $-1.711-2$ & 42 & 39 & $-1.126+1$ & 4.670 & $7.029-1$ & 43 & 16 & $1.296+0$ & -1.532 & $-8.229-2$ \\
\hline 40 & 20 & $-5.684-2$ & $-9.141+0$ & 5.888-2 & 42 & 38 & $-7.724+0$ & 3.098 & 4.869-1 & 43 & 15 & $-1.105-1$ & -9.159 & $-6.561-4$ \\
\hline 40 & 19 & $2.055+0$ & -1.773 & $-6.828-2$ & 42 & 37 & -5.588 & 2.195 & $3.378-1$ & 43 & 14 & $3.007+0$ & -2.125 & $-1.943-1$ \\
\hline 40 & 18 & 4.506-1 & -1.159 & $3.392-2$ & 42 & 36 & $-3.688+0$ & 1.322 & $2.222-1$ & 43 & 13 & $-5.600-1$ & -6.371 & $1.436-2$ \\
\hline 40 & 17 & $1.213+0$ & $-1.415+1$ & $-1.848-2$ & 42 & 35 & 5.925 & -5.051 & $-3.778-2$ & 43 & 12 & $-8.501-1$ & -5.982 & $4.200-2$ \\
\hline 40 & 16 & $-4.861-1$ & $-6.458+0$ & $7.859-2$ & 42 & 34 & $1.730+0$ & -1.008 & $-1.170-1$ & 43 & 11 & $-2.034+0$ & -1.387 & 1.153-1 \\
\hline 40 & 15 & $1.490+0$ & -1.450 & $-4.077-2$ & 42 & 33 & 4.054 & -2.059 & $-2.491-1$ & 43 & 10 & 9.646-1 & -1.479 & $-5.415-2$ \\
\hline 40 & 14 & 8.669-1 & -1.155 & $-5.335-3$ & 42 & 32 & $4.548+0$ & -2.357 & $-2.731-1$ & 43 & 9 & $-3.981-1$ & -9.910 & $3.115-2$ \\
\hline 40 & 13 & $1.812+0$ & $-1.501+1$ & $-6.737-2$ & 42 & 31 & $6.863+0$ & -3.328 & $-4.204-1$ & 43 & 8 & $-2.032+0$ & -3.399 & $1.264-1$ \\
\hline 40 & 12 & $-2.688+0$ & $3.723+0$ & $1.980-1$ & 42 & 30 & $5.904+0$ & -3.089 & $-3.436-1$ & 43 & 7 & $-4.558+0$ & 6.586 & $2.766-1$ \\
\hline 40 & 11 & $-1.780+0$ & $-2.844-1$ & $1.452-1$ & 42 & 29 & $8.460+0$ & -4.195 & $-5.002-1$ & 43 & 6 & $-8.317+0$ & 2.136 & 5.011-1 \\
\hline 40 & 10 & $-1.009+0$ & $-4.183+0$ & $1.048-1$ & 42 & 28 & $8.905+0$ & -4.435 & $-5.212-1$ & 43 & 5 & $-5.816+0$ & 1.014 & $3.548-1$ \\
\hline 40 & 9 & $-4.124+0$ & 8.125 & $2.938-1$ & 42 & 27 & 6.445 & -3.429 & $-3.792-1$ & 43 & 4 & $-6.122+0$ & 1.196 & $3.611-1$ \\
\hline 40 & 8 & $-2.869+0$ & 2.153 & $2.241-1$ & 42 & 26 & $8.043+0$ & -4.222 & $-4.582-1$ & 43 & 3 & $-4.728+0$ & 5.809 & $2.780-1$ \\
\hline 40 & 7 & $-4.245+0$ & $7.158+0$ & $3.100-1$ & 42 & 25 & $7.076+0$ & -3.805 & $-4.082-1$ & 43 & 2 & $-3.958+0$ & $1.907+0$ & $2.375-1$ \\
\hline 40 & 6 & $-5.603+0$ & $1.218+1$ & $3.919-1$ & 42 & 24 & $5.059+0$ & -3.032 & $-2.824-1$ & 43 & 1 & $-7.824+0$ & 1.896 & 4.531-1 \\
\hline 40 & 5 & $-3.939+0$ & $4.131+0$ & $3.031-1$ & 42 & 23 & $5.486+0$ & -3.226 & $-3.079-1$ & 43 & 0 & $-7.991+0$ & 2.030 & $4.565-1$ \\
\hline 40 & 4 & $-6.418+0$ & $1.327+1$ & 4.591-1 & 42 & 22 & $6.752+0$ & -3.814 & $-3.747-1$ & 44 & 44 & $-5.640-1$ & 1.369 & 5.794-1 \\
\hline 40 & 3 & $-6.648+0$ & $1.438+1$ & $4.664-1$ & 42 & 21 & $3.405+0$ & -2.489 & $-1.683-1$ & 44 & 43 & $-7.954+0$ & 2.877 & 7.045-1 \\
\hline 40 & 2 & $-4.377+0$ & $4.545+0$ & $3.351-1$ & 42 & 20 & $2.344+0$ & -1.994 & $-1.133-1$ & 44 & 42 & $-9.406+0$ & 3.566 & 6.691-1 \\
\hline 40 & 1 & $-5.083+0$ & $8.067+0$ & $3.663-$ & 42 & 19 & $3.088+0$ & -2.355 & $-1.498-1$ & 44 & 41 & $-1.164+1$ & 4.412 & 7.505-1 \\
\hline 40 & 0 & $-6.382+0$ & $1.331+1$ & $4.421-1$ & 42 & 18 & $1.429+0$ & -1.579 & $-6.124-2$ & 44 & 40 & $-1.198+1$ & 4.458 & $7.670-1$ \\
\hline 41 & 41 & -9.198-1 & $6.385+0$ & 4.994-1 & 42 & 17 & $3.072+0$ & $-2.259+$ & $-1.596-1$ & 44 & 39 & $-1.141+1$ & $4.134+1$ & 7.081-1 \\
\hline 41 & 40 & $-1.208+1$ & $4.395+1$ & $9.611-1$ & 42 & 16 & $3.295+0$ & $-2.306+$ & $-1.752-1$ & 44 & 38 & $-1.184+1$ & $4.267+1$ & 7.298-1 \\
\hline 4 & 39 & $-1.593+1$ & $6.500+1$ & $1.035+0$ & 42 & 15 & $1.183+0$ & -1.325 & $-6.135-2$ & 44 & 37 & $-8.649+0$ & 2.870 & $5.232-1$ \\
\hline 41 & 38 & $-1.054+1$ & $4.399+1$ & $6.707-1$ & 42 & 14 & $1.951+0$ & $-1.617+1$ & $-1.097-1$ & 44 & 36 & $-4.665+0$ & 1.227 & $2.730-1$ \\
\hline 41 & 37 & $-6.531+0$ & $2.699+1$ & 4.107-1 & 42 & 13 & $1.451+0$ & $-1.401+1$ & $-7.901-2$ & 44 & 35 & $-3.471+0$ & $7.588+0$ & $1.874-1$ \\
\hline 41 & 36 & $-3.801+0$ & $1.529+1$ & $2.381-1$ & 42 & 12 & $8.564-1$ & $-1.202+1$ & $-4.038-2$ & 44 & 34 & $-1.565-1$ & $-6.556+0$ & $-1.982-2$ \\
\hline 41 & 35 & $-1.177+0$ & $3.663+0$ & $7.732-2$ & 42 & 11 & 9.626-1 & $-1.326+1$ & $-3.926-2$ & 44 & 33 & $-1.446+0$ & $-1.992+0$ & $6.174-2$ \\
\hline 41 & 34 & $1.895+0$ & $-1.003+1$ & $-1.067-1$ & 42 & 10 & $-1.122+0$ & $-5.608+0$ & $9.380-2$ & 44 & 32 & $5.633+0$ & $-3.163+1$ & $-3.625-1$ \\
\hline 41 & 33 & $4.840+0$ & $-2.318+1$ & $-2.772-1$ & 42 & 9 & $-2.039+0$ & $-2.013+0$ & $1.461-\overline{1}$ & 44 & 31 & $3.860+0$ & $-2.530+1$ & $-2.557-1$ \\
\hline 41 & 32 & $4.361+0$ & $-2.159+1$ & $-2.538-1$ & 42 & 8 & $-5.209+0$ & $1.077+1$ & $3.350-1$ & 44 & 30 & $6.402+0$ & $-3.588+1$ & $-4.090-1$ \\
\hline 41 & 31 & $5.607+0$ & $-2.782+1$ & $-3.222-1$ & 42 & 7 & $-4.701+0$ & $8.179+0$ & $3.057-1$ & 44 & 29 & $5.349+0$ & $-3.170+1$ & $-3.536-1$ \\
\hline 41 & 30 & $6.508+0$ & $-3.283+1$ & $-3.639-1$ & 42 & 6 & $-6.544+0$ & $1.455+1$ & $4.259-1$ & 44 & 28 & $6.400+0$ & $-3.780+1$ & $-3.955-1$ \\
\hline
\end{tabular}

Table 2: Curve fit coefficients of VT transitions rates (continued) 


\begin{tabular}{|c|c|c|c|c|c|c|c|c|c|c|c|c|c|c|}
\hline$\nu_{i}$ & $\nu_{f}$ & a1 & $\mathrm{a} 2$ & a3 & $\nu_{i}$ & $\nu_{f}$ & a1 & $\mathrm{a} 2$ & a.3 & $\nu_{i}$ & $\nu_{f}$ & a1 & $\mathrm{a} 2$ & a.3 \\
\hline 44 & 27 & 6.273 & -3.719 & $-3.980-1$ & 45 & 33 & $-3.869+0$ & 3.516 & $6-1$ & 46 & 40 & 5.570 & -2.868 & $-3.956-1$ \\
\hline $\begin{array}{l}44 \\
44\end{array}$ & $\begin{array}{l}21 \\
26\end{array}$ & $7.134+0$ & $\begin{array}{l}-5.119+1 \\
-4.080+1\end{array}$ & $\begin{array}{l}-5.900-1 \\
-4.543-1\end{array}$ & 45 & $\begin{array}{l}50 \\
32\end{array}$ & $\begin{array}{l}-0.009+0 \\
-1009+0\end{array}$ & $\begin{array}{r}3.010+ \\
-8.868+\end{array}$ & $2.200-1$ & 46 & 39 & 6.781 & -3.51 & -4.819 \\
\hline $\begin{array}{l}44 \\
44\end{array}$ & 25 & $\begin{array}{l}1.134+0 \\
3.031+0\end{array}$ & $\begin{array}{l}-4.000 \\
-2.48\end{array}$ & $\begin{array}{l}-4.040-1 \\
-2010-1\end{array}$ & 45 & $\begin{array}{l}52 \\
31\end{array}$ & ${ }^{-1.009+0}$ & $\begin{array}{l}-0.000+0 \\
-1499+1\end{array}$ & 5.233-2 & 46 & 38 & 1.094 & -5.572 & -6.888 \\
\hline $\begin{array}{l}44 \\
44\end{array}$ & 24 & $\begin{array}{l}3.031+0 \\
3.959+0\end{array}$ & $\begin{array}{l}-2.483+1 \\
-2.845+1\end{array}$ & $\begin{array}{l}-2.010-1 \\
-2.622-1\end{array}$ & $\begin{array}{l}45 \\
45\end{array}$ & $\begin{array}{l}51 \\
30\end{array}$ & $\begin{array}{r}0.998-1 \\
-4815+0\end{array}$ & $-1.499+1$ & $-6.781-2$ & 46 & 37 & 1.152 & -6.134 & -7078 \\
\hline 44 & $\begin{array}{l}24 \\
23\end{array}$ & $6.394+0$ & $\begin{array}{l}-2.040+1 \\
-3.896+1\end{array}$ & $-4.0225-1$ & 45 & 50 & $-4.815+0$ & $0.031+0$ & $2.012-1$ & 46 & 36 & 7.862 & -4.861 & $-4.710-1$ \\
\hline 44 & 22 & +0 & $\begin{array}{l}-3.090 \\
-3.374\end{array}$ & $\begin{array}{l}-4.000-1 \\
-3.276-1\end{array}$ & 40 & $\begin{array}{l}29 \\
28\end{array}$ & & -2.210 & $1.359-1$ & 46 & 35 & 7.064 & -4.768 & $-4055-1$ \\
\hline 44 & 21 & $185.5+0$ & $\begin{array}{l}-0.014 \\
-2.070\end{array}$ & $-1.260-1$ & 45 & 28 & -1 & -1 & $3.477-2$ & 46 & 34 & $5.687+0$ & -4.372 & 181 \\
\hline $\begin{array}{l}44 \\
44\end{array}$ & $\begin{array}{l}21 \\
20\end{array}$ & $\begin{array}{l}1.855+0 \\
4.335+0\end{array}$ & -2.070 & $\begin{array}{l}-1.300-1 \\
-2824-1\end{array}$ & 45 & 27 & 65 & -1.57 & $-2.192-2$ & 46 & 33 & 6.431 & -4.980 & 10 \\
\hline 44 & 19 & $\begin{array}{l}4.335+0 \\
3.237+0\end{array}$ & -3.113 & - $2.824-1$ & 45 & 26 & $052-$ & -9.258 & $7.456-2$ & 46 & 32 & -157 & -1788 & .0. \\
\hline 44 & 18 & 3.2 & $\begin{array}{l}-2.645+ \\
-2861+\end{array}$ & $-2.218-1$ & 45 & 25 & 328 & 5.533 & 2.907-1 & 46 & 31 & -6556 & 2349 & $1.491-1$ \\
\hline $\begin{array}{l}44 \\
44\end{array}$ & $\begin{array}{l}10 \\
17\end{array}$ & 3. & $-2.861+$ & $-2.526-1$ & 45 & 24 & $2.254+$ & $-2.640-$ & -1.593-1 & 46 & 30 & -132 & 2.697 & $8.505-1$ \\
\hline $\begin{array}{l}44 \\
44\end{array}$ & 16 & 2.42 & $-1.377+$ & $-5.188-2$ & 45 & 23 & $-3.185+0$ & $-4.005+$ & $1.587-1$ & 46 & 20 & -1.67 & 4.261 & 039 \\
\hline 44 & 10 & $2.931+$ & $-2.490+1$ & $-2.080-1$ & 45 & 22 & $-2.900-1$ & $-1.612+$ & $-1.355-2$ & 46 & 28 & -1.4 & 3143 & 047 \\
\hline 44 & 15 & $-5.958-1$ & $-9.615+0$ & $-5.667-3$ & 45 & 21 & $-2.656+0$ & $-6.524+0$ & $1.280-1$ & 16 & 20 & 1715 & 4.223 & .047 \\
\hline 42 & 14 & $2.299+0$ & $-1.985+1$ & $-2.011-1$ & 45 & 20 & 8.739-1 & $-1.976-$ & $-1.042-1$ & 40 & 26 & -1.10 & 5.22 .0 & 068 \\
\hline 44 & 13 & $-4.449-1$ & $-9.850+0$ & $-1.872-2$ & 45 & 19 & 5.624-1 & $-1.890-$ & $-8.575-2$ & 40 & 20 & -1.92 & 0.010 & 8.3 \\
\hline 44 & 12 & $-1.045+0$ & $-6.695+0$ & $6.723-3$ & 45 & 18 & $-7.978-1$ & $-1.443+$ & $1.268-2$ & 40 & 25 & -2.00 & 0.440 & \\
\hline 44 & 11 & $1.890-1$ & $-1.246+1$ & $-6.245-2$ & 45 & 17 & $-2.483+0$ & -6.312 & $9.442-2$ & 40 & 24 & & & 1 \\
\hline 44 & 10 & $1.367+0$ & $-1.855+$ & $-1.177-1$ & 45 & 16 & $-4.962+0$ & 4.860 & $2.305-1$ & 46 & 23 & -2 & 8.275 & 607 \\
\hline 44 & 9 & $1.631+0$ & $-1.965+$ & $-1.364-1$ & 45 & 15 & 6.239 & $-1.740+$ & -1 119-1 & 46 & 22 & -2 & 6.080 & 296 \\
\hline 44 & 8 & $-1.416+0$ & $-7.979+0$ & $4.577-2$ & 45 & 14 & $-2486+0$ & -4154 & $6.506-2$ & 46 & 21 & $34:$ & 7.042 & $1.368+0$ \\
\hline 44 & 7 & $-7.298+0$ & $1.646+$ & $3.852-1$ & 45 & 13 & $-2.534+0$ & -3.222 & 5 & 46 & 20 & -2.051 & 5.657 & 213 \\
\hline 44 & 6 & $-2.434+0$ & $-4.815+0$ & $1.043-1$ & 45 & 12 & $2.670+0$ & -2.547 & $\begin{array}{l}0.010-2 \\
2.4201\end{array}$ & 46 & 19 & -1.864 & 5.081 & $072+0$ \\
\hline 44 & 5 & $-7.527+0$ & $1.599+$ & 4.006-1 & 45 & 11 & 2.010 & $\begin{array}{l}-2.0 \\
-2\end{array}$ & $\begin{array}{l}-2.499-1 \\
-2.295-1\end{array}$ & 46 & 18 & -2.17 & 6.272 & 1.260 \\
\hline 44 & 4 & $-7.526+0$ & $1.458+1$ & $4.168-1$ & 45 & 10 & $3.251+0$ & -2.864 & $-2.22 .0-1$ & 46 & 17 & -2.26 & 6.933 & 1.283 \\
\hline 44 & 3 & $-5.091+0$ & $4.728+0$ & $2.612-1$ & 45 & 9 & $\begin{array}{r}0.201 \\
-1.725+\end{array}$ & -8.185 & $\begin{array}{r}-2.140-1 \\
2078-2\end{array}$ & 46 & 16 & -1.35 & 3.113 & 37 \\
\hline 44 & 2 & $-9.400+0$ & 2.200 & $5.216-1$ & 45 & 8 & & $\begin{array}{l}-0.100 \\
-2123\end{array}$ & $2.018-2$ & 46 & 15 & -2.106 & 6.400 & 1.163 \\
\hline 44 & 1 & -9.242 & 2.253 & $4.932-1$ & 45 & 7 & -1.29 & -1.120 & $-1.900-1$ & 46 & 14 & -1.441 & 3.748 & $7.539-1$ \\
\hline 44 & $\stackrel{1}{0}$ & -1.179 & 3.302 & $6.494-1$ & 45 & 6 & $\begin{array}{l}-1.29 .0+0 \\
-4.565+0\end{array}$ & $-1.032-$ & $-1.002-2$ & 46 & 13 & -2.109 & 6.612 & $1.138+0$ \\
\hline 45 & 45 & $-1.667+0$ & $1.266+$ & $5.645-1$ & 45 & 5 & $-4267-1$ & $\begin{array}{l}1.450 \top \\
-1635+\end{array}$ & $\begin{array}{l}1.901-1 \\
-5\end{array}$ & 46 & 12 & -1.61 & 4.52 & 8.40 \\
\hline 45 & 44 & $-6.557+0$ & $2.461+$ & $6.307-1$ & 45 & 4 & $-2487+0$ & $-9.388+$ & $\begin{array}{r}-5.501-2 \\
8010\end{array}$ & 46 & 11 & -1.452 & 3.847 & 7.456 \\
\hline 45 & 43 & $-1.140+1$ & 4.249 & $7.782-1$ & 45 & $\begin{array}{l}4 \\
3\end{array}$ & -2.429 & $\begin{array}{l}-9.000 \\
-1.02 \%\end{array}$ & $0.919-2$ & 46 & 10 & -1.291 & 3.212 & 6.369 \\
\hline 45 & 42 & $-1.235+1$ & $4.362+1$ & $8.546-1$ & 45 & 2 & $-4.831-2$ & $\begin{array}{l}-1.022+ \\
-2.090+\end{array}$ & $5.000-2$ & 46 & 9 & -9.698 & 1.847 & $4.534-1$ \\
\hline 45 & 41 & $-1.155+1$ & $3.921+1$ & $7.657-1$ & 45 & 1 & $-7.743+0$ & 1.143 & $\begin{array}{r}-5.300-2 \\
3971-1\end{array}$ & 46 & 8 & -3.634 & -6.609 & 7.543 \\
\hline 45 & $\begin{array}{l}41 \\
40\end{array}$ & $-9.457+0$ & $2.986+1$ & $6.397-1$ & 45 & $\stackrel{1}{0}$ & $-6064+0$ & 6.140 & 3.9 $71-1$ & 46 & 7 & -1.617 & 4.404 & $8.37^{\circ}$ \\
\hline 45 & 39 & $-8.282+0$ & 2.497 & $5.300-1$ & 46 & 46 & -0. & & $2.720-1$ & 46 & 6 & -1.633 & 4.502 & $8.238-1$ \\
\hline 45 & 38 & $-9.360+0$ & $2.913+1$ & $5.869-1$ & 46 & 45 & -4.60 & 1. & 0.48 & & 5 & (Oח & .784 & $1.073+0$ \\
\hline 45 & 37 & $-7.502+0$ & & $4.582-1$ & 46 & 44 & $-8.383+0$ & $\frac{1.03}{2}$ & 6.01 & 4 & 4 & -3 & 1.03 & 1.669 \\
\hline 45 & 36 & $-6212+0$ & $1.456+1$ & $3833-1$ & 46 & 43 & $-7570+0$ & $\begin{array}{l}2.149+ \\
2610+1\end{array}$ & $0.010-1$ & 46 & 3 & -7.430 & 8.064 & $\therefore 71$ \\
\hline 45 & 35 & -0.212 & 1.4 & $3.504-1$ & 46 & $\begin{array}{l}40 \\
42\end{array}$ & -2.05 & $\begin{array}{l}2.019+ \\
8102+\end{array}$ & $4.550-1$ & 46 & 2 & -8.864 & 1.587 & 35 \\
\hline 45 & 34 & $-4.41^{\circ}$ & 7.018 & $2.486-1$ & 46 & 41 & 1018 & $8.192+0$ & $1.419-1$ & 4 & 1 & -1.030 & 1.995 & $4.368-1$ \\
\hline & & & & & & & & & & 4 & 0 & -3.801 & 1.309 & $2.147+0$ \\
\hline
\end{tabular}

Table 2: Curve fit coefficients of VT transitions rates (end) 


\section{References}

${ }^{1}$ J. G. Kim and I. D. Boyd, "State-resolved master equation analysis of thermochemical nonequilibrium of nitrogen," Chemical Physics, vol. 415, pp. 237-246, 2013.

${ }^{2}$ M. Panesi, R. L. Jaffe, D. W. Schwenke, and T. E. Magin, "Rovibrational internal energy transfer and dissociation of $\mathrm{N}_{2}$ $\left({ }^{1} \Sigma_{g}^{+}\right)-\mathrm{N}\left({ }^{4} S_{u}\right)$ system in hypersonic flows," Journal of Chemical Physics, vol. 138, no. 4, p. 044312, 2013.

${ }^{3}$ J. G. Kim and I. D. Boyd, "Thermochemical nonequilibirum modeling of electronically excited molecular oxygen," in 11th AIAA/ASME Joint Thermophysics and Heat Transfer Conference, AIAA paper 2014-2963, 2014.

${ }^{4} \mathrm{~F}$. Esposito and M. Capitelli, "The relaxation of vibrationally excited $\mathrm{O}_{2}$ molecules by atomic oxygen," Chemical Physics Letters, vol. 443, no. 4, pp. 222-226, 2007.

${ }^{5} \mathrm{M}$. Quack and J. Troe, "Complex formation in reactive and inelastic scattering: Statistical adiabatic channel model of unimolecular processes III," Berichte der Bunsengesellschaft für physikalische Chemie, vol. 79, no. 2, pp. 170-183, 1975.

${ }^{6}$ A. Varandas and A. Pais, "A realistic double many-body expansion (DMBE) potential energy surface for ground-state $\mathrm{O}_{3}$ from a multiproperty fit to ab initio calculations, and to experimental spectroscopic, inelastic scattering, and kinetic isotope thermal rate data," Molecular Physics, vol. 65, pp. 843-860, Nov 1988.

${ }^{7}$ D. Steele, E. R. Lippincott, and J. T. Vanderslice, "Comparative study of empirical internuclear potential functions," Reviews of Modern Physics, vol. 34, no. 2, p. 239, 1962.

${ }^{8}$ G. Herzberg, Molecular Spectra and Molecular Structure. van Nostrand, 1957.

${ }^{9}$ R. C. Millikan and D. R. White, "Systematics of vibrational relaxation," Journal of Chemical Physics, vol. 39, no. 12, p. $3209,1963$.

${ }^{10}$ K. L. Wray, "Shock-tube study of the vibrational relaxation of nitric oxide," Journal of Chemical Physics, vol. 36, no. 10, pp. 2597-2603, 1962.

${ }^{11}$ D. Eckstrom, "Vibrational relaxation of shock-heated $\mathrm{N}_{2}$ by atomic oxygen using the ir tracer method," Journal of Chemical Physics, vol. 59, no. 6, pp. 2787-2795, 1973.

${ }^{12}$ J. Breen, R. Quy, and G. Glass, "Vibrational relaxation of $\mathrm{O}_{2}$ in the presence of atomic oxygen," The Journal of Chemical Physics, vol. 59, no. 1, pp. 556-557, 1973.

${ }^{13}$ J. H. Kiefer and R. W. Lutz, "The effect of oxygen atoms on the vibrational relaxation of oxygen," in Symposium (International) on Combustion, vol. 11, pp. 67-76, Elsevier, 1967.

${ }^{14}$ G. M. Burnett and A. M. North, "Transfer and storage of energy by molecules," Zeitschrift fur Physikalische Chemie, vol. 228,1969 .

${ }^{15}$ E. L. Breig, "Statistical model for the vibrational deactivation of molecular by atomic oxygen," Journal of Chemical Physics, vol. 51, no. 10, p. 4539, 1969.

${ }^{16}$ C. Lee and H.-R. Kim, "A classical trajectory study of $\mathrm{O}+\mathrm{O}_{2}$ collision," Chemical Physics Letters, vol. 233, no. 5, pp. 658-664, 1995.

${ }^{17}$ D. Andrienko and I. D. Boyd, "Vibrational relaxation and dissociation in collisions with parent atom," in sumitted to the 45th AIAA Thermophysics Conference, AIAA, 2015.

${ }^{18}$ R. B. Bernstein, "Atom-molecule collision theory; a guide for the experimentalist," in Atom-molecule Collision Theory; a Guide for the Experimentalist, vol. 1, 1979.

${ }^{19}$ C. W. Eaker, G. C. Schatz, N. De Leon, and E. J. Heller, "Fourier transform methods for calculating action variables and semiclassical eigenvalues for coupled oscillator systems," Journal of Chemical Physics, vol. 81, no. 12, pp. 5913-5919, 1984.

${ }^{20}$ L. Lapidus and J. H. Seinfeld, Numerical Solution of Ordinary Differential Equations. Elsevier, 1971.

${ }^{21}$ A. Gross and G. D. Billing, "Isotope effects on the rate constants for the processes $\mathrm{O}_{2}+\mathrm{O} \rightarrow \mathrm{O}+\mathrm{O}_{2}$ and $\mathrm{O}_{2}+\mathrm{O}+$ $\mathrm{Ar} \rightarrow \mathrm{O}_{3}+\mathrm{Ar}$ on a modified ground-state potential energy surface for ozone," Chemical Physics, vol. 217, pp. 1-18, 1997.

${ }^{22}$ C. Park, "Rotational relaxation of $\mathrm{N}_{2}$ behind a strong shock wave," Journal of Thermophysics and Heat Transfer, vol. 18, no. 4, pp. 527-533, 2004.

${ }^{23}$ C. Park, "Review of chemical-kinetic problems of future nasa missions. I - Earth entries," Journal of Thermophysics and Heat Transfer, vol. 7, pp. 385-398, Jan 1993.

${ }^{24}$ F. Esposito, I. Armenise, G. Capitta, and M. Capitelli, "O $+\mathrm{O}_{2}$ state-to-state vibrational relaxation and dissociation rates based on quasiclassical calculations," Chemical Physics, vol. 351, pp. 91-98, Jul 2008.

${ }^{25}$ L. Ibraguimova, A. Sergievskaya, and O. Shatalov, "Dissociation rate constants for oxygen at temperatures up to 11000 k," Fluid Dynamics, vol. 48, no. 4, pp. 550-555, 2013.

${ }^{26}$ L. Landau and E. Teller, "Theory of sound dispersion," Physik. Z. Sowjetunion, vol. 10, p. 34, 1936.

${ }^{27}$ G. Colonna, F. Esposito, and M. Capitelli, "The role of rotation in state-to-state vibrational kinetics," in 9 th AIAA/ASME Joint Thermophysics and Heat Transfer Conference, AIAA Paper 2006-3423, 2006.

${ }^{28}$ J. G. Kim, O. J. Kwon, and C. Park, "Master equation study and nonequilibrium chemical reactions for $\mathrm{H}+\mathrm{H}_{2}$ and $\mathrm{He}$ $+\mathrm{H}_{2}$," Journal of Thermophysics and Heat Transfer, vol. 23, pp. 443-453, Jul 2009.

${ }^{29}$ M. Capitelli, C. M. Ferreira, A. I. Osipov, and B. F. Gordiets, Plasma Kinetics in Atmospheric Gases. Springer, 2000.

${ }^{30}$ M. Camac, "O 2 vibration relaxation in oxygen-argon mixtures," Journal of Chemical Physics, vol. 34, no. 2, pp. 448-459, 1961.

${ }^{31}$ S. Bauer and S. Tsang, "Mechanisms for vibrational relaxation at high temperatures," Physics of Fluids, vol. 6, no. 2, pp. 182-189, 1963. 